\title{
Coupling from the Photosphere to the Chromosphere and the Corona
}

\author{
S. Wedemeyer-Böhm • A. Lagg • Å. Nordlund
}

Received: 26 August 2008 / Accepted: 14 September 2008 / Published online: 8 November 2008

(C) Springer Science+Business Media B.V. 2008

\begin{abstract}
The atmosphere of the Sun is characterized by a complex interplay of competing physical processes: convection, radiation, conduction, and magnetic fields. The most obvious imprint of the solar convection and its overshooting in the low atmosphere is the granulation pattern. Beside this dominating scale there is a more or less smooth distribution of spatial scales, both towards smaller and larger scales, making the Sun essentially a multi-scale object. Convection and overshooting give the photosphere its face but also act as drivers for the layers above, namely the chromosphere and corona. The magnetic field configuration effectively couples the atmospheric layers on a multitude of spatial scales, for instance in the form of loops that are anchored in the convection zone and continue through the atmosphere up into the chromosphere and corona. The magnetic field is also an important structuring agent for the small, granulation-size scales, although (hydrodynamic) shock waves also play an important role-especially in the internetwork atmosphere where mostly weak fields prevail. Based on recent results from observations and numerical simulations, we attempt to present a comprehensive picture of the atmosphere of the quiet Sun as a highly intermittent and dynamic system.
\end{abstract}

Keywords Sun · Photosphere · Chromosphere · Corona · Convection · Magnetohydrodynamics $\cdot$ Radiative transfer

S. Wedemeyer-Böhm ( $₫)$

Institute of Theoretical Astrophysics, University of Oslo, Oslo, Norway

e-mail: svenwe@astro.uio.no

A. Lagg

MPI für Sonnensystemforschung, Katlenburg-Lindau, Germany

e-mail: lagg@ mps.mpg.de

Å. Nordlund

Niels Bohr Institute, University of Copenhagen, Copenhagen, Denmark

e-mail: aake@nbi.dk 


\section{Introduction}

Observations of the solar atmosphere reveal a wealth of different phenomena, which occur over an extended range of different temporal and spatial scales. This is not surprising, considering the fact that already basic parameters such as gas density and temperature span many orders of magnitude, from the convection zone below the photosphere to the corona. At a first look, it may thus appear rather hopeless to construct an overall picture that can account for all the phenomena. At a closer look, however, many connections between apparently independent phenomena can be found, ultimately implying a multitude of couplings through the atmosphere. In addition, there seems to be a hierarchical arrangement of approximately selfsimilar convective motions, with the granulation pattern embedded in increasingly larger meso- and supergranulation patterns.

The key to a comprehensive picture of the solar atmosphere thus lies in relaxing too strict and oversimplified concepts, even when they are didactically nicer than the reality. The solar atmosphere should not be seen as a static stack of layers but rather as intermittent domains that are dynamically coupled together. One example is magnetic flux structures (or "flux tubes") fanning out with a wine-glass geometry. Such regular building blocks put certain constraints on the implied atmospheric structure, which can make it difficult to fit in other observational findings. Accepting that magnetic field structures are far less regular offers room for a more generally valid comprehensive picture. This trend became more and more obvious during the recent years, both from the observational and theoretical side (see, e.g., Carlsson 2007; Gudiksen 2006; Hansteen 2007; Judge 2006; Rutten 2007; Steiner 2007 and many more).

The advantages of a relaxed picture can be seen from the example of the quiet Sun chromosphere above internetwork regions, which in itself is a complex and intriguing phenomenon (see, e.g., Judge 2006; Rutten 2006; De Pontieu et al. 2004; Lites et al. 1999 and many more). Despite tremendous progress, there are still many open questions concerning its structure, dynamics and energy balance. Recent observations now prove-beyond any doubt - the chromosphere to be a highly dynamic and intermittent layer. The internetwork chromosphere is the product of a dynamic interplay of shock waves and magnetic fields. This picture, which was already suggested by many earlier investigations, offers a key to resolve some apparent contradictions that lead to much confusion in the past. A prominent example concerns the observation of carbon monoxide (see, e.g., Ayres 2002), which now can be explained as an integral part of a dynamic and intermittent atmosphere (Wedemeyer-Böhm and Steffen 2007; Wedemeyer-Böhm et al. 2005a, 2006). And still the chromosphere cannot be investigated without also taking into account the layers above and below. The shock waves, which are so essential at least for the lowest, weak-field parts of the chromosphere, are generated in the layers below, while significant amounts of mass and energy are exchanged between the chromosphere and the corona above. Obviously, the whole atmosphere must be seen as an integral phenomenon.

In the following sections, we report on a selection of results from observations and numerical simulations, which will help us put together an updated, revised view of the structure of the quiet Sun atmosphere.

\section{The Sun-A Multi-Scale Object}

An overarching point in this discussion is the fact that the Sun is fundamentally a multiscale object. This is a major difficulty for modeling and understanding, since it requires (computationally expensive) modeling over a large range of scales. 
But the Sun also displays aspects of self-similarity and scale invariance in several respects, which on the other hand helps a lot. To illustrate the self-similarity, Fig. 1a shows temperature patterns in horizontal planes in a large scale simulation of solar convection (Zhao et al. 2007), and Fig. 1b shows patterns of vertical velocities from the same simulation. The temperature patterns show very intermittent cold structures, embedded in a background of horizontally nearly constant temperature (images of entropy would look essentially identical, with near-constancy also in the vertical direction). The set of panels also shows that the pattern scales increase systematically with depth.

Figure $1 \mathrm{~b}$, on the other hand, which displays vertical velocity on a color scale that changes from yellow to blue with sign (with a narrow band of grey for velocities near zero) gives a completely different impression. With this rendering choice one can see that, at least from a morphological point of view, the patterns at different depths are quite similar. Displaying in this way, signed velocity reveals that the sharply defined dark (cold) patterns in Fig. 1a indeed correspond to the strongest downward velocities, but that there are also relatively broad areas of much milder downflows. This shows that, as the ascending gas is forced (by mass conservation) to overturn, it does so at first gently, then to finally be accelerated more strongly by the positive feedback that comes from merging with colder gas from above.

At the visible surface the horizontal velocity patterns from various depths are superposed. This happens because the depth dependencies of the large scale horizontal velocity patterns are rather weak; at least over depth intervals small compared to their horizontal extents. On the other hand, as illustrated by Fig. 1, the dominant scale becomes smaller for layers increasingly close to the surface. As the amplitudes of these smaller scales are larger, they mask the presence of the larger scale patterns, whose presence, however, can still be revealed, e.g., with Fourier analysis or with low-pass filtering. The hierarchy is illustrated in a side view in Fig. 2. The combination of streamlines and colors illustrate how near-surface, small scales fluctuations are carried along in larger scale flows.

The hierarchy of scales displayed reveals no particular preferred scale above the granular one; the transition to larger and larger scales with depth is smooth. A direct way to illustrate this from observations is to use power spectra of solar velocities, as observed with SOHO/MID (Georgobiani et al. 2007). Figure 3 shows velocity (mainly horizontal) as a function of size, produced by filtering the velocity power observed by MDI into sonic and sub-sonic parts. The velocity spectrum displayed is produced by then taking the square root of the velocity power times wave number; this is a quantity - a velocity spectrum - that nicely illustrates the dependence of velocity amplitude on size. Note that there is very little (less than a factor two) extra power at scales traditionally associated with supergranulation, and that there is a smooth and increasing distribution of velocity amplitude across the "meso-granulation" range to granulation scales. The same behavior is found in large scale numerical simulations (Georgobiani et al. 2007).

Supergranulation patterns can be brought forward by averaging over either time or space; the dashed line in Fig. 3 shows the effect of a 24-hour time average. A low-pass wavenumber spatial filter has a similar effect; it cuts away the larger amplitudes at smaller scales and exposes aspects of the underlying larger scale pattern. The relatively distinct appearance of a supergranulation scale network in magnetically related diagnostics indicates that the transport and diffusion of magnetic field structures at the solar surface results in what is effectively a low-pass wavenumber filter.

As shown by Fig. 4 it is practically impossible to tell the difference between velocity patterns on different scales, once they are filtered to effectively the same resolution. As illustrated by Stenflo and Holzreuter $(2002,2003)$ magnetic field patterns and distributions also show a degree of self-similarity. 
a)
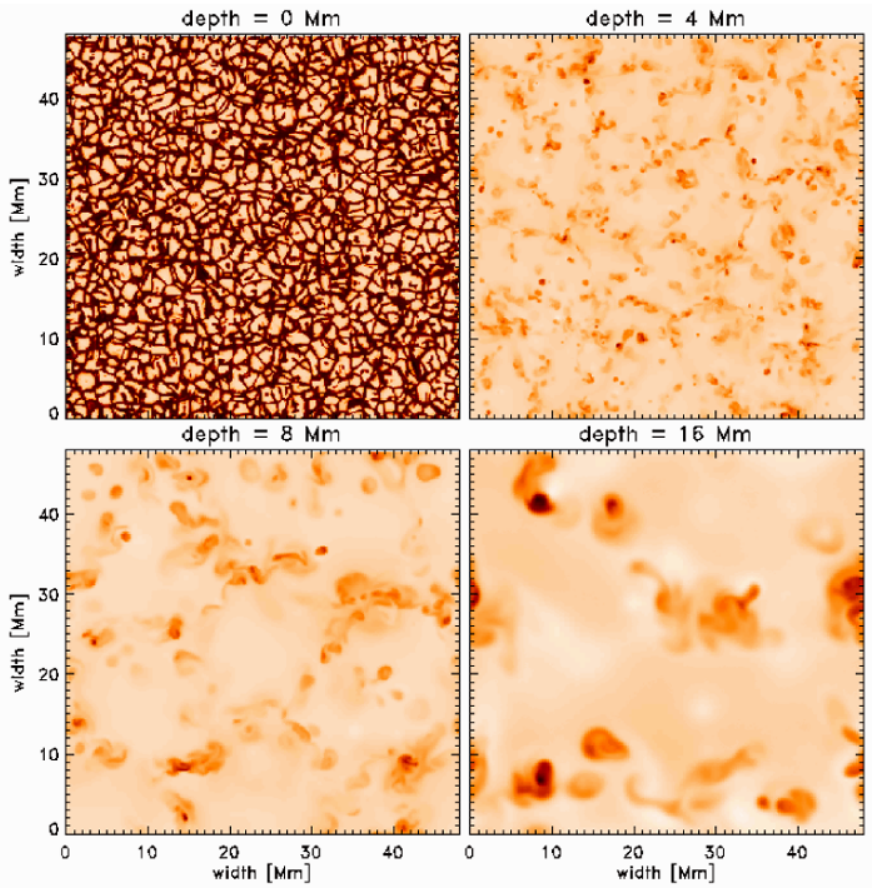

b)

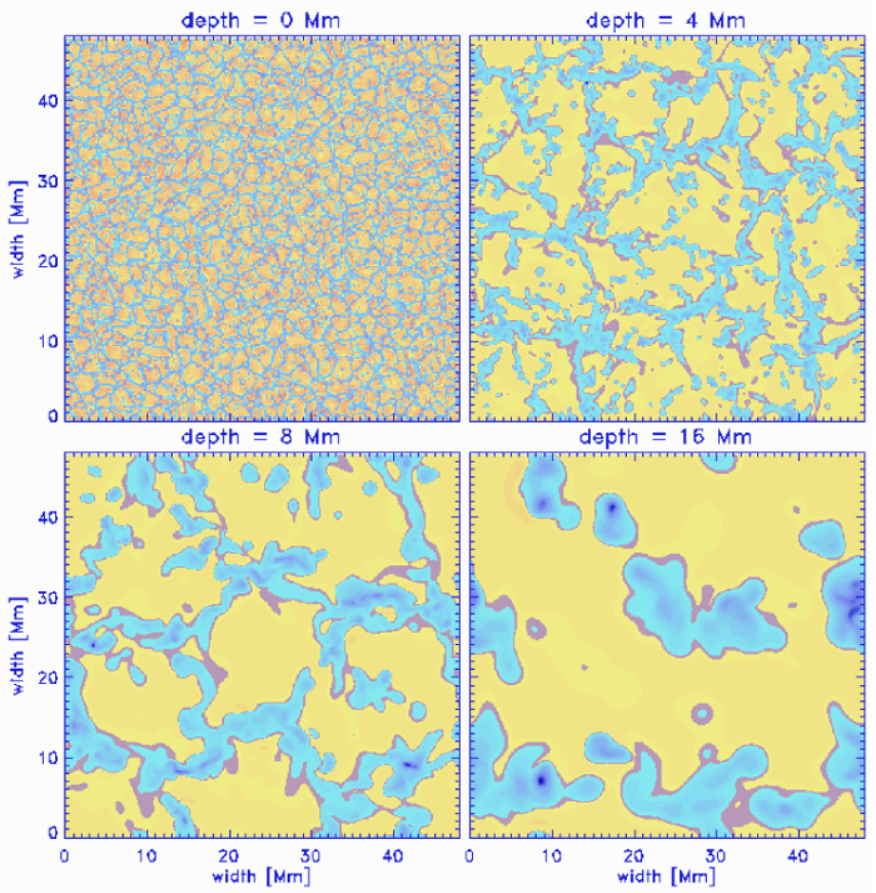

Fig. 1 Large scale solar convection $(48 \mathrm{Mm} \times 48 \mathrm{Mm} \times 20 \mathrm{Mm}$ ). a Temperature (top four panels) and b velocity (bottom four panels) patterns at four different depths. Temperature is shown on a linear scale. Velocities are rendered with positive (downward) values blue and negative (upward) velocities yellow. A narrow band near zero velocity is rendered in grey 


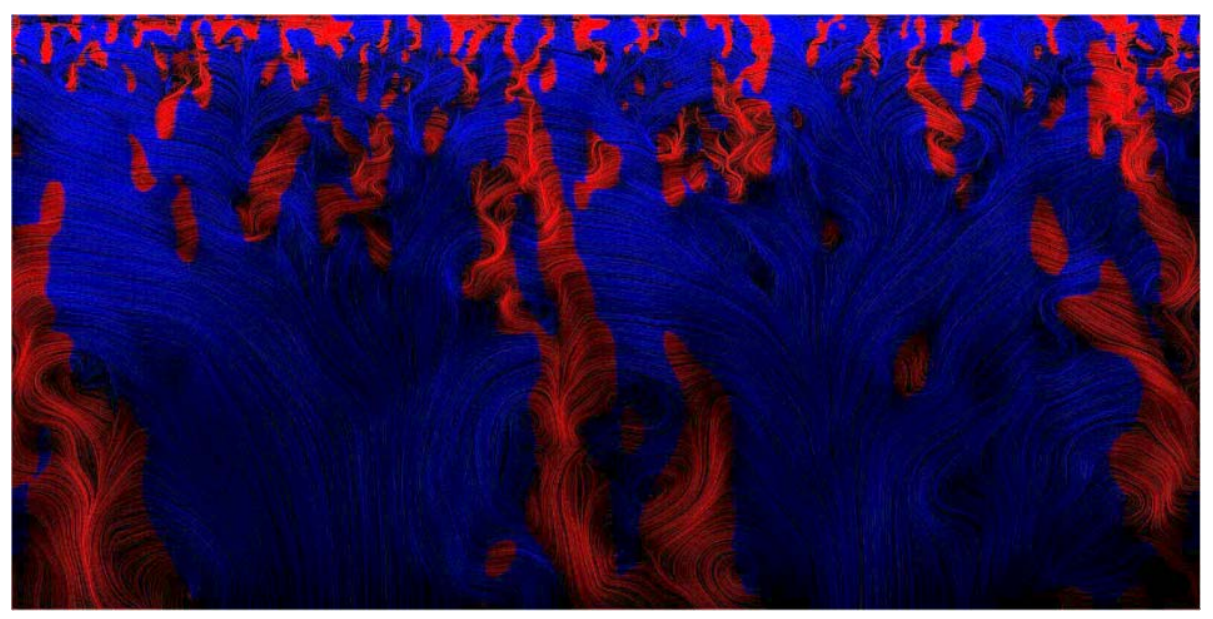

Fig. 2 A side view of a $48 \mathrm{Mm} \times 48 \mathrm{Mm} \times 20 \mathrm{Mm}$ simulation, showing velocity streamlines, with brightness increasing with increasing magnitude. Up- and down-flows are rendered in blue and red, respectively

Fig. 3 Solar velocity spectrum: A subsonic $\left(7 \mathrm{~km} \mathrm{~s}^{-1}\right)$ filter has been used to separate the velocity into oscillatory (grey) and convective (black) components. The dashed line shows the convective component resulting from first taking a $24 \mathrm{~h}$ average. Adapted from Georgobiani et al. (2007)

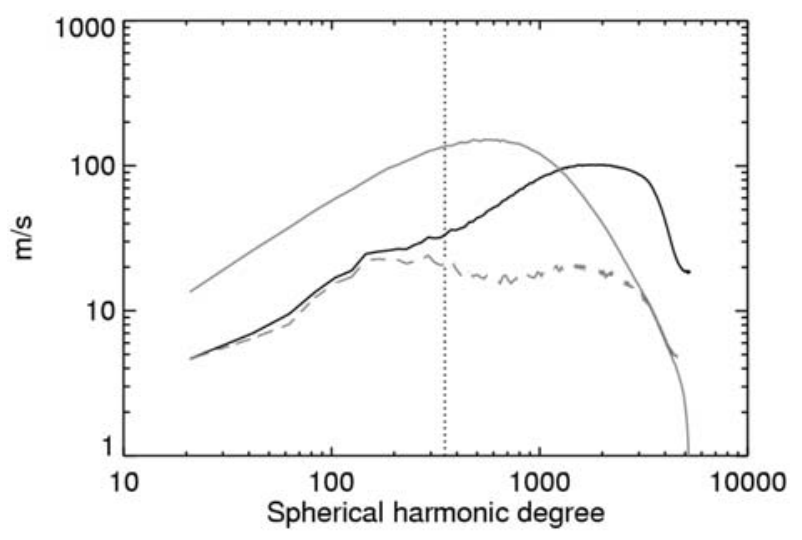

The magnetic energy equation

$$
\frac{\partial}{\partial t}\left(\frac{\mu B^{2}}{2}\right)=-\nabla \cdot(\mathbf{E} \times \mu \mathbf{B})-\mathbf{v} \cdot(\mathbf{j} \times \mathbf{B})-Q_{\text {Joule }}
$$

illustrates that balance of the magnetic energy at each depth is achieved by Lorentz force work (by the flow on the field) being used to balance magnetic dissipation, with net magnetic energy transported up or down by the Poynting flux, $\mathbf{E} \times \mu \mathbf{B}$. As shown by the work of Vögler and Schüssler (2007) and Steiner et al. (2008) the actual direction of net transport is systematically downwards, at least below the solar surface. It appears likely that there is net dynamo action at each depth in the convection zone, with net magnetic energy delivered to the next layer down. This naturally leads to a pile up near the bottom of the convection zone. The downward transport, which is known from direct studies (Tobias et al. 1998, 2001; Dorch and Nordlund 2001), is often referred to as "turbulent pumping", and is associated with the asymmetry between up and downflows (illustrated in Fig. 1b). 
Fig. 4 Solar horizontal velocities observed with SOHO/MDI. A patch some distance away from solar center has been compensated for projection effects and filtered to effective resolutions that differ by factors of 2 . Which is which?
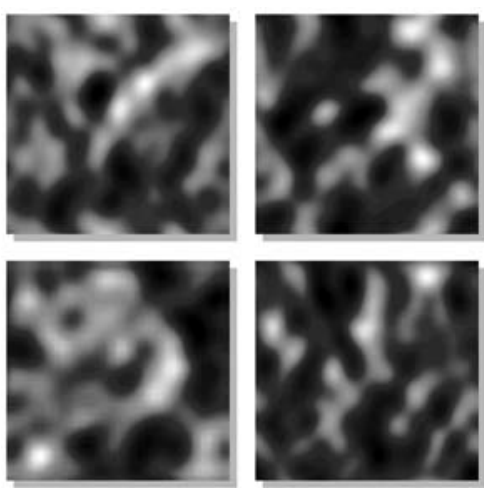

On the largest scales (largest depths), and only there, differential rotation enables a large scale global dynamo action, with patterns clearly controlled by being stretched out by differential rotation. Buoyancy eventually pushes the fields back up.

Another evidence for self-similarity comes from the power law behavior of flare energy distribution as a function of time. This behavior is also recovered in numerical simulations of 3D magnetic reconnection (Galsgaard and Nordlund 1996). These are signs that magnetic reconnection occurs in a multi-scale hierarchy, where magnetic dissipation at large magnetic Reynolds number (low resistivity) creates a hierarchy. Large scale structures generate subsidiary small scale structures, which do it again (on shorter time scales) and again, until the spatial scales are small enough to support the dissipation.

Note the remarkable and wonderful argument, made already by Parker a long time ago, which shows that driven magnetic dissipation must, if anything, increase with decreasing resistivity-quite contrary to naive expectations. This has been verified in numerical experiments by at least three different groups (Galsgaard and Nordlund 1996; Hendrix et al. 1996; Dmitruk and Gómez 1999).

The chromosphere and corona are likely to be heated in much the same way, as is illustrated by the well known flux-flux relations between coronal and chromospheric diagnostic. It is hard to even avoid, as in models of coronal heating there is a tendency of dumping much more energy in the chromosphere as a side effect (Gudiksen and Nordlund 2002, 2005b).

As pointed out by Phil Judge: The chromosphere is not a mess; the upper chromosphere looks nearly force-free like the corona, whereas the lower chromosphere is less force-free. Complexity comes from both the temperature and density. A central question is: What drives the flows (particularly the cool upflows)?

Semi-realistic models of coupling of the horizontal photospheric velocity field to the corona were first computed by Gudiksen and Nordlund (2005b), who showed that a correctly normalized photospheric (model) velocity field injects sufficient power into the corona to create and maintain coronal temperatures (cf. Fig. 5).

The mechanism is, in the absence of explicit flux emergence, essentially the 'braiding mechanism' introduced by Parker $(1972,1981,1983)$. The heating is quite intermittent and drives up- and down-flows along magnetic field lines into and out of the corona. Emulated TRACE images and animations show qualitative agreement with observations (Peter et al. 2004). Silicon IV, for example, picks up cooling condensations (cf. Fig. 6). In addition, spectral lines formed at different temperatures show semi-quantitative agreement of the dependency with depth of their Doppler shifts and mean emission measures (cf. Figs. 7 and 9, Peter et al. 2006). The differential emission measure is a 'fingerprint' type diagnos- 


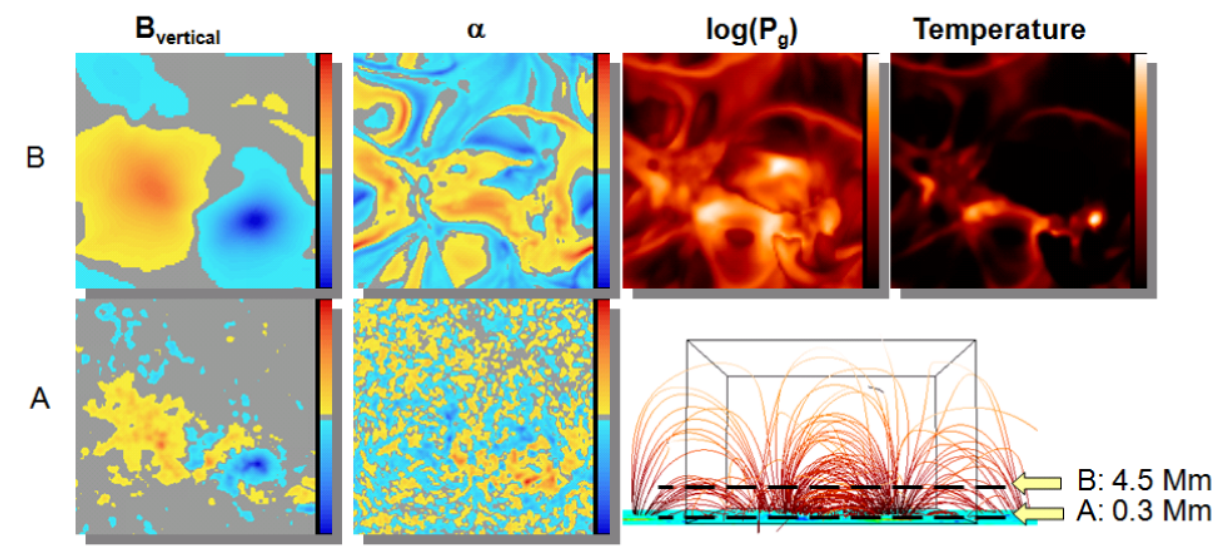

Fig. 5 The panels show the vertical magnetic field (far left), the ratio (often referred to with the symbol $\alpha$ ) of the magnitude of the electric current along the magnetic field to the magnitude of the magnetic field itself (left), the gas pressure (right) and the log. temperature (far right). The positions of the cutting planes are indicated in the inset at bottom right. Adapted from Gudiksen and Nordlund (2005b)

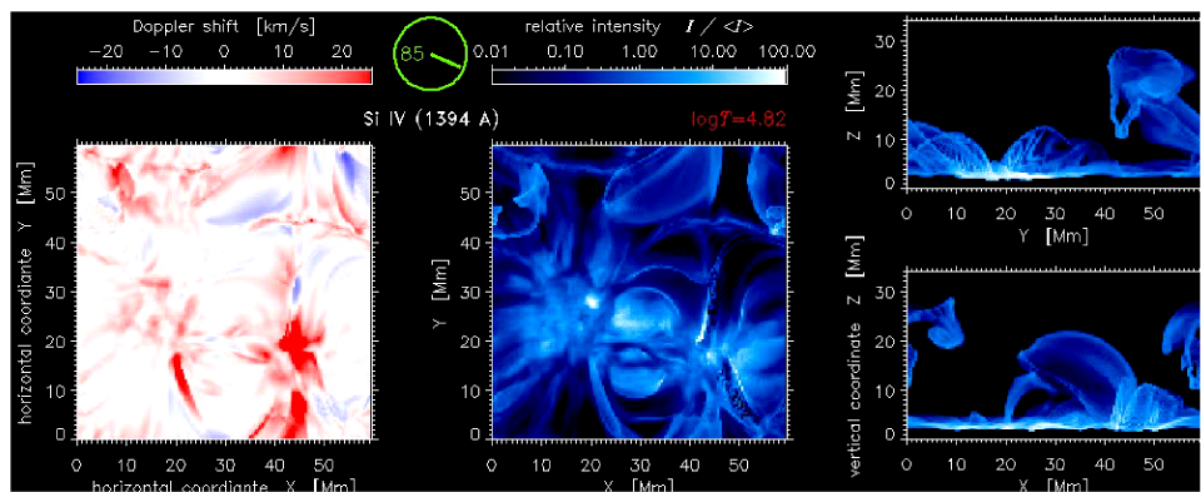

Fig. 6 The Si IV $1394 \AA$ Aoppler shift (left) and emission measure (mid) as they would be observed from above, and the emission measure projected along the Y-axis (right). Positive (downward) Doppler shifts are in red and negative Doppler shifts are in blue. Adapted from Peter et al. (2004)

tic, in much the same way as spectral line asymmetries are for photospheric spectral lines (Dravins et al. 1981; Dravins and Nordlund 1990; Asplund et al. 2000a, 2000b). Subsequently there has been much progress due to the work of the Oslo group (Hansteen and Gudiksen 2005; Hansteen et al. 2006, 2007; Martínez-Sykora et al. 2008)—cf. also the discussion in Sect. 4.3.

\section{Observations-Measuring the Magnetic Field in the Solar Atmosphere}

The $\mathrm{H} \alpha$ line core images in Figs. 7h and 8 show a well-known but still barely understood and intricate picture: fibrils that spread from regions of enhanced magnetic field strength, occasionally connecting to neighboring regions or apparently fading in between (see e.g. Rutten 2007). The structure gradually changes as one goes from line center into the wings, 

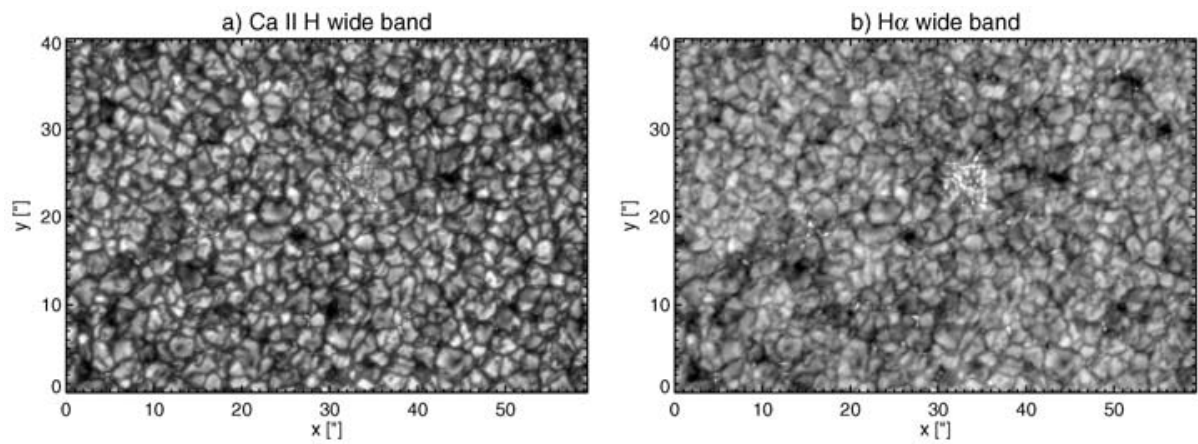

c) Ca II H wing $(396.5 \mathrm{~nm})$
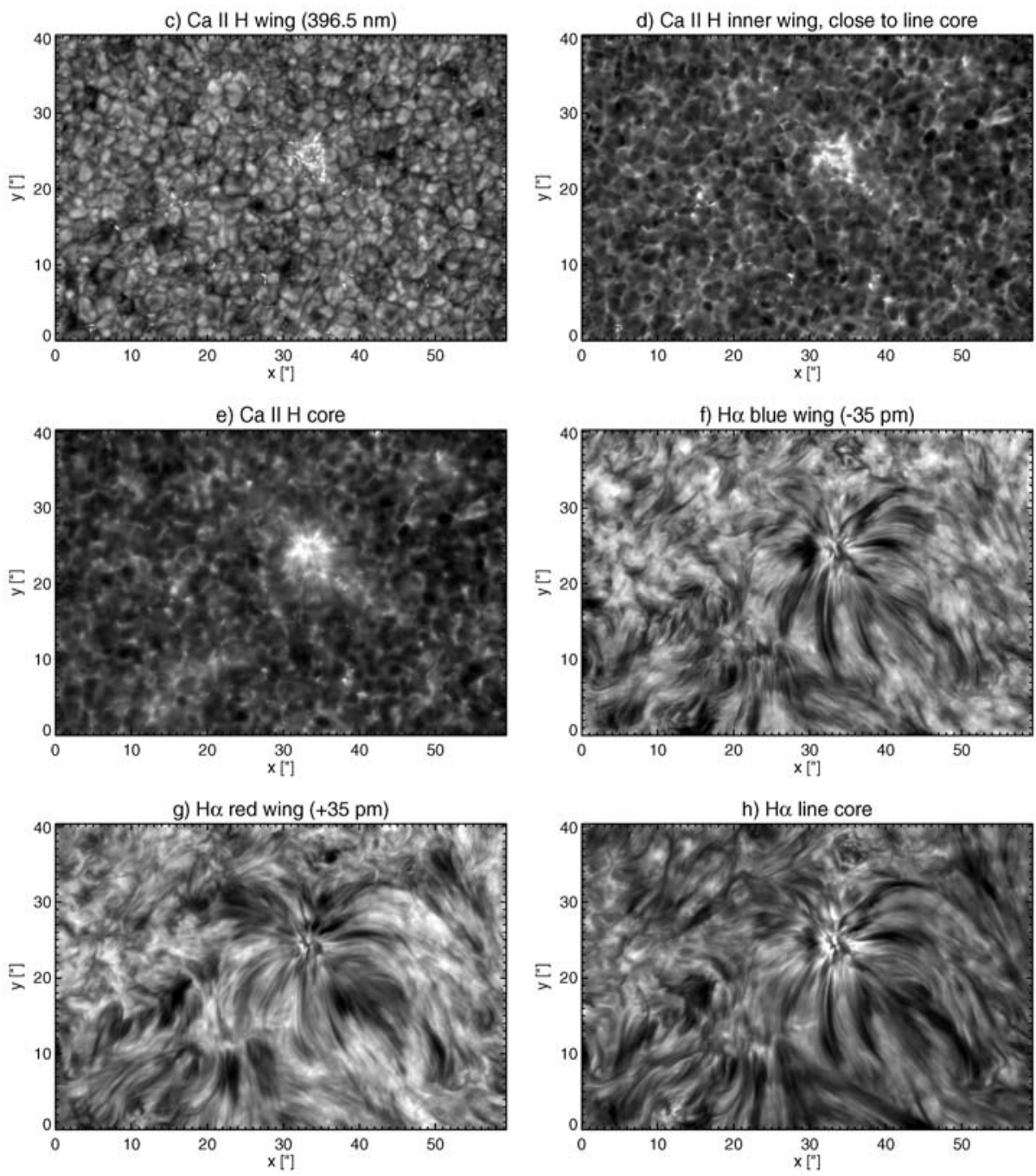

Fig. 7 Observations of a quiet Sun region close to disc-centre: a Ca II H wide band, b $\mathrm{H} \alpha$ wide band image (FWHM $0.8 \mathrm{~nm}$ ), c Ca II H wing $(396.5 \mathrm{~nm})$, d Ca II H inner wing (close to line core), e Ca II H core, f H $\alpha$ blue wing at $-35 \mathrm{pm}, \mathbf{g ~ H} \alpha$ red wing $+35 \mathrm{pm}, \mathbf{h ~ H} \alpha$ line core. The observations were carried out with the Swedish 1-m Solar Telescope (SST). Data courtesy: L. Rouppe van der Voort (University of Oslo) 


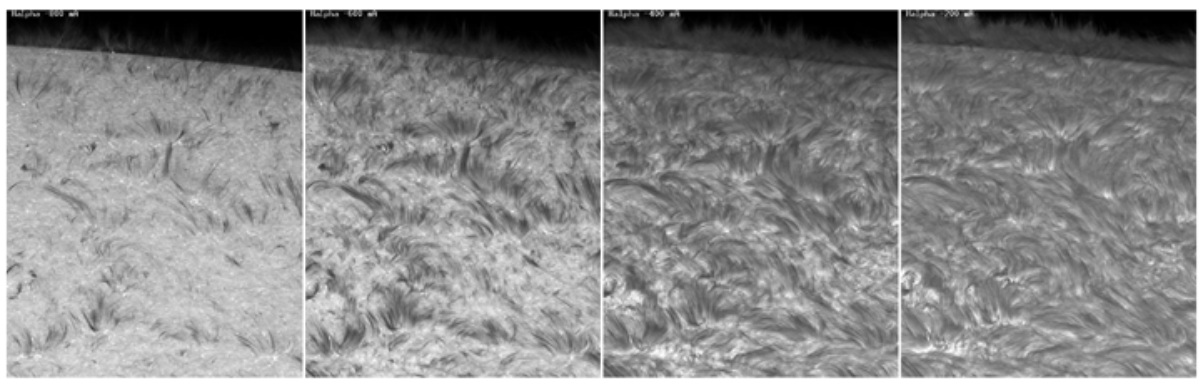

Fig. $8 \mathrm{H} \alpha$ fine structure at $-800,-600,-400,-200 \mathrm{~m} \AA$ from line center recorded with the Dutch Open Telescope (DOT) on October 4, 2005 (taken from Rutten 2007). Cell-spanning fibrils are visible around line center (right). The decreasing line opacity in the blue wing of the line opens the view to the solar photosphere, intercepted by dark fibrils resulting from the Doppler shift of the line core

as the corresponding intensity is due to lower layers. Finally, a mesh-like background pattern shines through in the internetwork regions. It is most likely due to reversed granulation in the middle photosphere with some possible contributions from the low chromosphere. The gradual change of the pattern in $\mathrm{H} \alpha$ with wavelength gives some clues about the atmospheric structure, in particular the magnetic field in the chromosphere (the "canopy" field), and definitely shows us that the photosphere and chromosphere are coupled via magnetic fields on medium to large spatial scales and via fields and shock waves on the small scales.

Therefore, the understanding of the coupling between photosphere and corona is intimately connected to the measurement of the chromospheric magnetic field. The following subsections exemplify the difficulties of chromospheric magnetic field measurements and present promising approaches to determine the vector magnetic field of the chromosphere.

\subsection{Improving Magnetic Field Extrapolations}

Measuring the magnetic field in the photosphere has a long tradition (Hale 1908). After 100 years of solar magnetic field measurements, the level of sophistication, both in terms of instrumentation and in analysis technique, has reached a very high level of maturity. $\mathrm{Nu}-$ merous telescopes, ground based and space born (e.g. GONG, MDI), investigate the global structure of the solar magnetic field on a routine basis. High resolution measurements allow the characterization of magnetic elements with a size in the $100 \mathrm{~km}$ range (e.g., SST-CRISP, Hinode SP). With HMI on SDO and the balloon-borne $1 \mathrm{~m}$ telescope Sunrise (launch: summer 2009) a major improvement in the determination of photospheric magnetic fields will be achieved in both directions - the global magnetic field configuration as well as the smallest scale structures down to a size of $25 \mathrm{~km}$.

The availability of such high quality photospheric vector magnetograms and the low plasma- $\beta$ in the chromosphere are the basic ingredients needed for reliable, force-free magnetic field extrapolations. Starting with Sakurai (1981) these extrapolations nowadays have reached a high level of sophistication (see reviews by Sakurai 1989; Amari et al. 1997; Wiegelmann 2008). To further improve the accuracy of the chromospheric magnetic field extrapolations additional information on the complex structure of the chromosphere must be taken into account. One of the most promising advances in this direction was proposed by Wiegelmann et al. (2008): the basic assumption for applying non-linear, force-free magnetic field extrapolations is the force-freeness of the photospheric vector magnetograms. Measured magnetograms do not fulfill this requirement, therefore a preprocessing of the 
measured data is required. Wiegelmann et al. (2008) developed a minimization procedure that yields a more chromosphere-like field by including the field direction information contained in, e.g., chromospheric $\mathrm{H} \alpha$ images. Including this information into the extrapolation algorithm significantly enhances the reliability of the extrapolations.

\subsection{Direct Measurements of the Chromospheric Magnetic Fields}

Measurement techniques for chromospheric magnetic fields have to overcome a variety of hurdles: (i) the plasma density is several orders of magnitude lower than in the photosphere, (ii) the energy transport is dominated by radiation, (iii) the magnetic field strength is on average lower than in the photosphere, and (iv) anisotropic illumination induces population imbalances between atomic sublevels that are modified by weak magnetic fields. The low plasma density leads to weak signals in the absorption (on-disk observations) or emission (off-limb observations) of spectral lines. The absorption signatures of chromospheric lines often show a strong photospheric contribution. Only highly spectrally resolved observations of the line core carry the chromospheric information. As a consequence of the low density, the simplifying assumption of local thermodynamic equilibrium breaks down. The interpretation of the observations is thus by far more involved than in the case of photospheric observations. Additionally, the low chromospheric magnetic field strengths weakens the Zeeman signals in spectral lines. Scattering polarization and its modification by the Hanle effect introduce an additional complication in the analysis of the polarization signal of spectral lines.

During the last decade major progress has been achieved in circumventing these hurdles. Radio observations are able to determine the magnetic field strength in and around active regions (see review by Lee 2007). Acoustic mapping techniques (Finsterle et al. 2004) use the reflection of high-frequency acoustic waves (mHz-range) from the region in the atmosphere where the gas pressure and the magnetic pressure are equal to reveal the structure of the magnetic canopy. The biggest leap in the direct determination of chromospheric magnetic fields was achieved by combining state of the art instrumentation for full Stokes polarimetry with recent progress in atomic physics. Bommier (1980), Landi Degl'Innocenti (1982), Stenflo and Keller (1997) and Trujillo Bueno et al. (2002) opened a new diagnostic window in solar physics: magnetic fields influence the strength and the direction of the linear polarization resulting from atomic or scattering polarization. This effect, discovered by Hanle (1991), allows the determination of the magnetic vector from Milligauss to several tens of Gauss, a range not accessible by Zeeman diagnostics.

The following sections describe examples of measurements in this new diagnostic window, focused around two of the most popular spectral lines for combined Hanle and Zeeman measurements: the triplet of $\mathrm{He} \mathrm{I} 10830 \AA$ and the $\mathrm{He} \mathrm{I} \mathrm{D}_{3} 5876 \AA$ multiplet. The formation of these lines requires ionization of para-He by ultraviolet radiation or collisions, followed by recombination to populate the lower sates of ortho-He. Since the main source for the ultraviolet radiation is the corona, these He I lines lacks almost any photospheric contribution. Additionally, they are (generally) optically thin and narrow, allowing the use of rather simple analysis techniques, like Milne-Eddington inversions of the radiative transfer equations (Solanki et al. 2003; Lagg et al. 2004, 2007). With the inversion code HAZEL (HAnle and ZEeman Light, see Asensio Ramos et al. 2008), involving the joint action of atomic level polarization and the Hanle and Zeeman effect in these lines, a standard tool for the analysis of Stokes spectra is now available. 


\subsubsection{Spicules}

Spicules are an ubiquitous phenomenon on the Sun. At any time, the number of these needle-like structures on the Sun is on the order of $4 \times 10^{5}$. These dynamic and short lived features (lifetimes typically 5-10 minutes) can be considered as magnetic tunnels through which the refueling of the coronal plasma takes place (Athay 2000). High cadence Hinode SOT observations in Ca II H (Okamoto et al. 2007; Suematsu et al. 2007) revealed details in terms of size and dynamics and led to the discovery of a new type of spicules (type II spicules, De Pontieu et al. 2007a) with shorter lifetimes (10-150 s), smaller diameters ( $<200 \mathrm{~km}$ compared to $<500 \mathrm{~km}$ for type I spicules), and shorter rise times. According to De Pontieu et al. (2007b), they (i) act as tracers for Alfvén waves with amplitudes of the order of 10 to $25 \mathrm{~km} \mathrm{~s}^{-1}$ and (ii) carry, in principle, enough energy to play an important role for the heating of the quiet Sun corona and for acceleration of the solar wind. See also Sect. 5.2.

Measurements of the magnetic field of spicules, both type I and type II, are essential for the understanding of this phenomenon. (Trujillo Bueno et al. 2005) were the first to directly demonstrate the existence of magnetized, spicular material. Full Stokes polarimetric data in the He I $10830 \AA$ A line, obtained with the Tenerife Infrared Polarimeter (Collados et al. 1999), were analyzed by solving the radiative transfer equation assuming an optically thick atmosphere. The application of a combined Hanle and Zeeman diagnostic revealed a magnetic field strength for the observed type I spicule of $10 \mathrm{G}$ and an inclination angle of $37^{\circ}$ at a height of $2000 \mathrm{~km}$ above the photosphere. The authors state that $10 \mathrm{G}$ is the typical field strengths for spicules at this height, but significantly stronger fields may also be present. This result agrees with the measurements from López Ariste and Casini (2005) using full Stokes polarimetry in the $\mathrm{He} \mathrm{I} \mathrm{D}_{3}$ line. They find field strengths not higher than $40 \mathrm{G}$ and a good correlation between the magnetic field orientation and the visible structure in $\mathrm{H} \alpha$ (see Fig. 9). An independent confirmation of these measurements was presented by Socas-Navarro and Elmore (2005) by using full Stokes observations from SPINOR (SpectroPolarimeter for INfrared and Optical Regions, at the Dunn Solar Telescope). Their multiline approach removes the dependence of the strength of the Hanle signals on the zero-field polarization produced by the scattering of anisotropic radiation in the higher atmosphere.

\subsubsection{Prominences and Filaments}

The spectacular eruptions of prominences and filaments and the resulting coronal mass ejections (CME) can cause sudden changes in the terrestrial magnetosphere. A typical CME releases an energy of $10^{25} \mathrm{~J}$ and $10^{12} \mathrm{~kg}$ of solar material into the interplanetary space (Harrison 1994). Before eruption, solar magnetic field holds this dense and relatively cool material in the hot coronal environment and supports it against the solar gravity for time periods as long as weeks. The knowledge of the magnetic field within these structures therefore is of great interest to understand the mechanisms leading to a possible eruption.

Casini et al. (2003) were the first to present magnetic maps of prominences using full Stokes polarimetry in the $\mathrm{He} \mathrm{I}_{3}$ line. Their results confirm previous measurements of the average field in prominences, ranging between 10 and $20 \mathrm{G}$ and oriented horizontally with respect to the solar surface. However, they also find the presence of organized structures in the prominence plasma embedded in magnetic fields that are significantly larger than average (50 G and higher). Merenda et al. (2007) extended this work to include the forward scattering case, applied it to a filament located at disk center and obtained the first magnetic maps of a filament. In this preliminary work they restricted their analysis to the saturated 

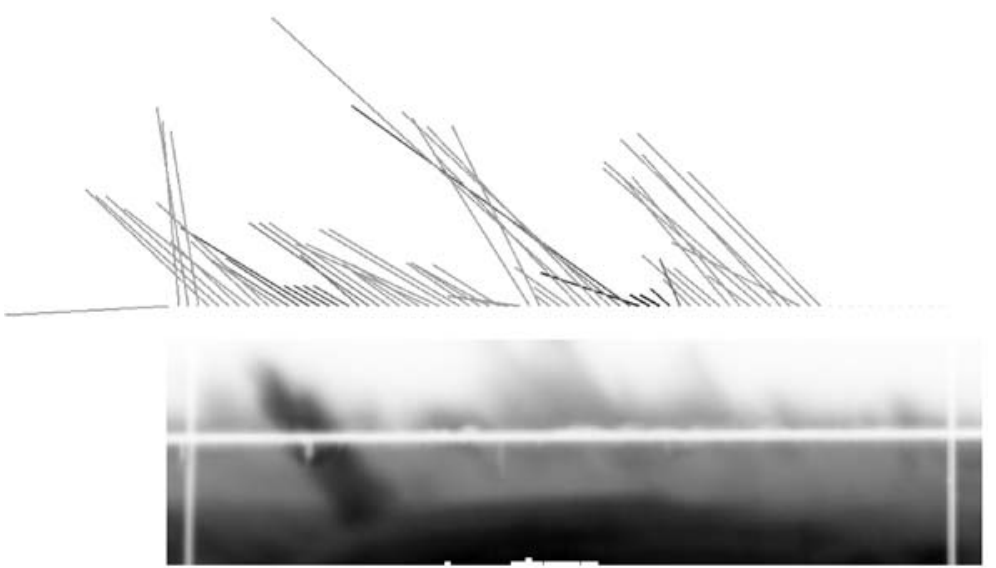

ASP Data. $\mathrm{D}_{3}$ spectropolarimetry: Operation 4 $5 / 28 / 2$ at 13:56:02 UT

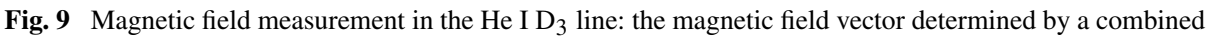
Hanle and Zeeman diagnostic traces the visible structures in the $\mathrm{H} \alpha$ slit-jaw image (bottom, adapted from López Ariste and Casini 2005)

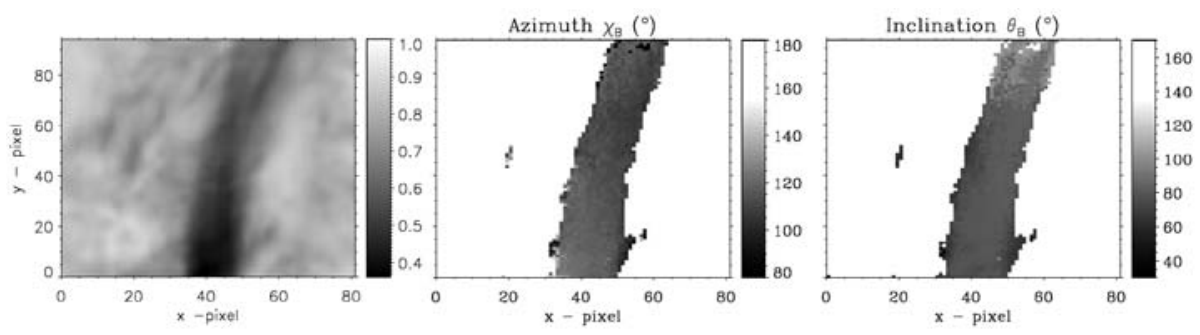

Fig. 10 Intensity image in the center of the He I $10830 \AA$ line and derived azimuthal and inclination angle of the magnetic field (adapted from Merenda et al. 2007)

Hanle effect regime between 10 and $100 \mathrm{G}$. Here the linear polarization is only sensitive to the direction of the magnetic field and does not change with intensity variations. The results are reliable maps for the azimuth and inclination angle for the magnetic field (see Fig. 10). In agreement with Casini et al. (2003) they find horizontal fields in the central part of the filament and a change of the azimuth according to the orientation of the main axis of the filament. In order to detect, for example, the small-scale and rapidly moving filaments mentioned in Sects. 4.2 and 5.3, significant improvements in signal to noise ratio and temporal resolution of polarimetric observations in this spectral line are required. The complex magnetic field and velocity structure of an erupting filament in the He I $10830 \AA$ line was analyzed by Sasso et al. (2007): besides the magnetic field topology they identify the presence of up to 5 different atmospheric components, distinguished by their velocities ranging from -50 to $+100 \mathrm{~km} \mathrm{~s}^{-1}$, within the resolution element of approximately $1.5^{\prime \prime}$. This measurement clearly demonstrates the fibrilar structure of the chromosphere (see also Lagg et al. 2007) and the need for higher spatial resolution measurements in this line. 


\subsubsection{Canopy}

Following previous work by W. Livingston, Gabriel (1976) introduced the term canopy to explain the emission measures of chromospheric and transition region UV lines. In the "classical" picture, the magnetic pressure wins over the gas pressure with increasing height, so that the magnetic flux concentrations rooted in the network expand and cover the internetwork cells with horizontal fields (see Sect. 5 for an updated view). Giovanelli and Jones (1982) and Jones and Giovanelli (1982) performed detailed studies of the magnetic canopy close to the limb by determining magnetograms using chromospheric spectral lines like the Ca II triplet at $8542 \AA$ or the $\mathrm{Mg} \mathrm{I} \mathrm{b}_{2}$ line at $5173 \AA$. These magnetograms are characterized by a polarity inversion line parallel to the limb, on either side surrounded by diffuse fields above the internetwork region (see Steiner and Murdin 2000 for a sketch of the magnetic configuration).

Especially during the last decade diagnostic tools involving the Hanle effect significantly improved the possibilities to characterize the canopy fields. Using spectropolarimetric data in the Sr II $4078 \AA$ line "Hanle histograms", showing the statistical distributions of the Hanle rotation and depolarization effects, Bianda et al. (1998) determined the magnetic field strength of horizontal, canopy-like fields to be in the range of 5 to $10 \mathrm{G}$. The first spatial mapping of Hanle and Zeeman (Stenflo et al. 2002) effect revealed details of canopy fields in a semi-quiet region measured close to the limb in the Na I $D_{1}-D_{2}$ system. The authors found direct evidence for horizontal magnetic fields, slightly stronger than the field strengths determined by Bianda et al. (1998) (25-35 G), that remain coherent over a spatial scale of at least three supergranules.

The concept of a magnetic canopy around sunspots and in active regions is well established. Over quiet regions, the formation of this layer of horizontal fields is matter of debate: Schrijver and Title (2003) showed that concentrations of magnetic flux in the network in the order of a few tens of $\mathrm{Mx} \mathrm{cm}^{-2}$ will destroy the classical, wineglass-shaped magnetic field topology. Such flux concentrations, suggested by simulations, were identified by Trujillo Bueno et al. (2004) in terms of ubiquitous tangled magnetic field with an average strength of $\approx 130 \mathrm{G}$, much stronger in the intergranular regions of solar surface convection than in the granular regions. A significant fraction of this hidden magnetic flux has now been clearly identified with the spectropolarimeter of the Hinode spacecraft (Lites et al. 2008). However, narrow-band $\left(0.1 \AA\right.$ ) observations in the Ca K line with a spatial resolution of $0.1^{\prime \prime}$ obtained with the Swedish Solar Telescope (SST) provide evidence that magnetic fibrils, originating from network flux concentrations, do span over a large distance above the quiet Sun network (see Figs. 7 and 11). Magnetic field measurements using the He I $10830 \AA$ line also indicate the presence of a uniform, horizontal magnetic field topology over the internetwork at mesogranular scales (Lagg and Merenda 2008). These measurements, presented in Fig. 12, were obtained with the Tenerife Infrared Polarimeter II (TIP-2) mounted behind the Vacuum Tower Telescope (VTT) on Tenerife (Collados et al. 2007) at a heliocentric angle of $49^{\circ}(\mu=\cos \Theta=0.65)$. Inversions involving the Hanle and Zeeman effects prove the presence of a horizontal "canopy" magnetic field on mesogranular scales with strengths of the order of 50 to $100 \mathrm{G}$, similar to the value of the averaged magnetic field of the underlying photosphere. Both, the recent narrow-band Ca K observations of (e.g., Pietarila et al. 2008; Rouppe van der Voort et al. 2005) and the magnetic field measurements (e.g., Lagg and Merenda 2008) seem to be in apparent contradiction to Schrijver and Title (2003), pointing out the necessity of a more detailed analysis on the validity of the concept of the magnetic canopy over quiet Sun regions. Nevertheless, the different finding can be fit into a common picture, when taking into account the sampled height ranges and a field topology, which is 


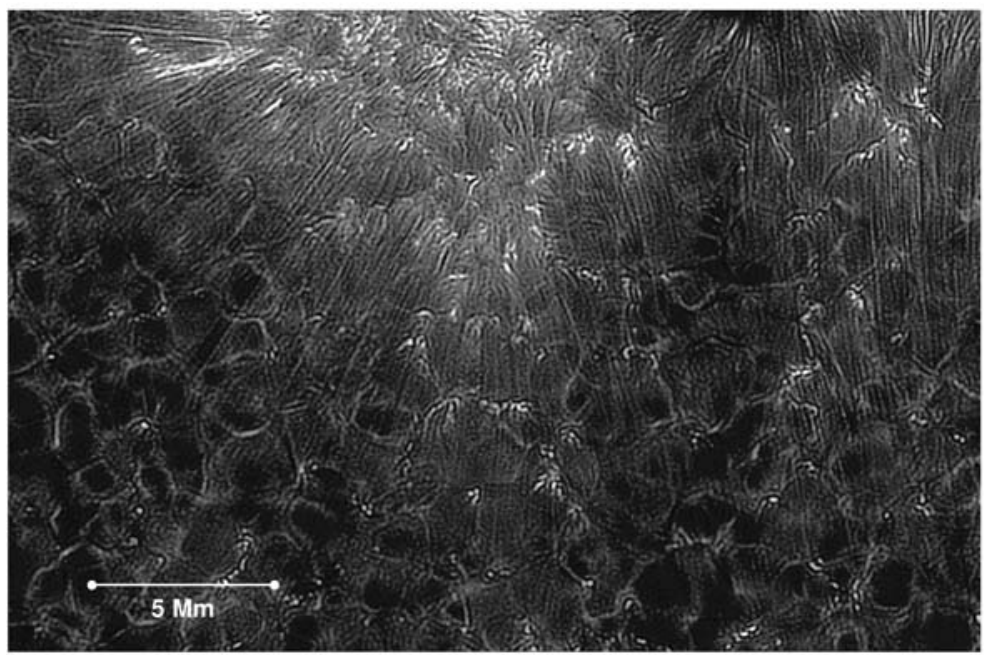

Fig. 11 Speckle-reconstructed, narrow band image (contrast-enhanced) of a plage region observed in the line core of Ca K using the SST (Pietarila et al. 2008, cf. Rouppe van der Voort et al. 2005). The Ca K fibrils extend over quiet Sun regions. The mesh-like background pattern is nevertheless dominated by the reversed granulation pattern in the middle photosphere (cf. Fig. 7)

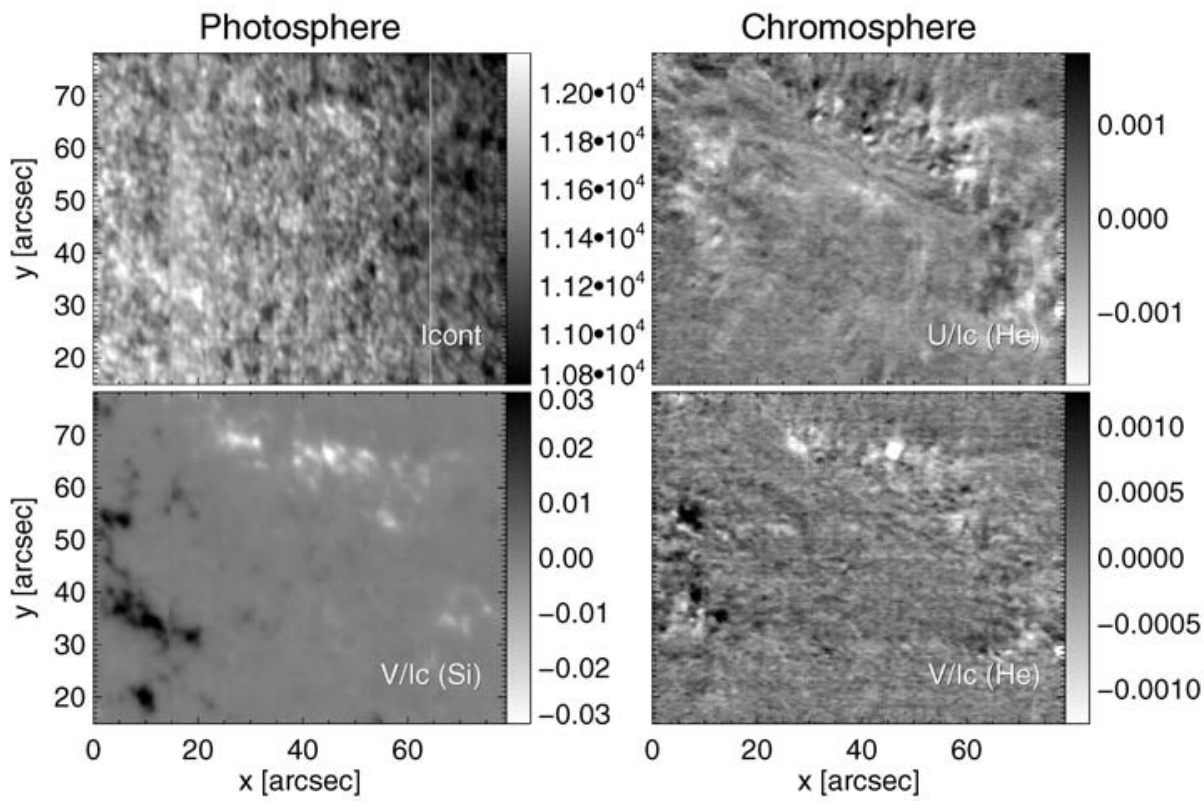

Fig. 12 Measurement of the magnetic field over a supergranular cell in the photosphere and the chromosphere (German Vacuum Tower Telescope, Tenerife Infrared Polarimeter 2, May 10, 2008): continuum close to the $10830 \AA$ line (top left), Stokes $V$ signal integrated over the red wing of the photospheric Si I $10827 \AA$ line (bottom left), Stokes $U$ and $V$ signal integrated over red wing of the chromospheric He I $10830 \AA$ line (top and bottom right, respectively). The chromospheric maps suggest the presence of magnetic structures organized on mesogranular scales within the supergranular cell outlined by the photospheric Stokes $V$ map 
more complex and entangled on small scales than usually assumed (see Sect. 5). The "classical" canopy might be in some ways a too simplified and thus potentially misleading concept.

\section{Numerical Simulations of the Quiet Sun}

\subsection{Internetwork Photosphere}

The solar granulation is now well reproduced by modern radiation (magneto-)hydrodynamical simulations. The contrast of continuum intensity or "granulation contrast" is often used for comparisons between observations and simulations. For many years, the contrast derived from observations were much lower than those found in numerical simulations. One reason is the often unknown but crucial effect of an optical instrument and the Earth atmosphere, resulting in a significant decrease of the granulation contrast. This problem can partially be overcome by using observations with space-borne instruments. Recent observations with the Broadband Filter Imager (BFI) of the Solar Optical Telescope (SOT) onboard the Hinode spacecraft now show higher contrast values. After application of a realistic point spread function (Wedemeyer-Böhm 2008; Danilovic et al. 2008), state-of-the-art numerical simulations indeed reproduce important characteristics of "regular" granulation.

The convective flows in and just above granule interiors advect magnetic field laterally towards the intergranular lanes, where the field is concentrated in knots and sheets with up to kilo-Gauss field strengths. In the granule interiors, usually only weak field remains, although in some situations flux concentrations of up to a few hundred Gauss can occur within the granules (Steiner et al. 2008). The latter finding is in agreement with the observations by Centeno et al. (2007) and Ishikawa et al. (2008). This process of "flux expulsion" has been known since early simulations (Galloway and Weiss 1981; Nordlund 1986). It is now an integral part of magnetoconvection simulations (see, e.g., Weiss et al. 1996; Stein and Nordlund 1998; Steiner et al. 1998; Schaffenberger et al. 2005; Vögler et al. 2005). The close-up from a simulation by Schaffenberger et al. (2005) in Fig. 13 illustrates the process. The magnetic field in the low photosphere is not only advected laterally. It is also lifted upwards and is concentrated above the reversed granulation layer at a height, which roughly corresponds to the classical temperature minimum in semi-empirical models (Fontenla et al. 1993). There, the convective overshooting effectively dies out and most of the upward directed flows above the granule interiors turn into lateral flows (Fig. 13). In the models by Wedemeyer et al. (2004), Wedemeyer (2003), the rms velocity amplitudes are smallest at these heights. The result is that the magnetic field is "parked" there and forms a mostly horizontally aligned field. It connects to the photospheric flux funnels, which spread out from the intergranular lanes below. The enclosed regions below, on the other hand, are virtually field-free with field strengths of possibly down to a few Gauss only (Steiner 2003). The field configuration around these granular voids was referred to as a dynamic "smallscale canopy" by Schaffenberger et al. (2005, 2006). Virtually field-free granule interiors are very common in their simulations. In the more recent simulations by Steiner et al. (2008), this phenomenon is also existent but less pronounced, although the field in the granular interiors is still much weaker than in the surrounding lanes. The main differences between these simulations is the average field strength $(10 \mathrm{G}$ and $20 \mathrm{G}$, resp.) and the injection of horizontal field at the lower boundary in the latter simulation. Obviously, the exact occurrence of smallscale canopies still depends on details of the simulations and thus needs to be checked by comparison with observations. The recent detection of so-called "horizontal inter-network fields" (HIFs) can be regarded as observational support for the small-scale field structure 

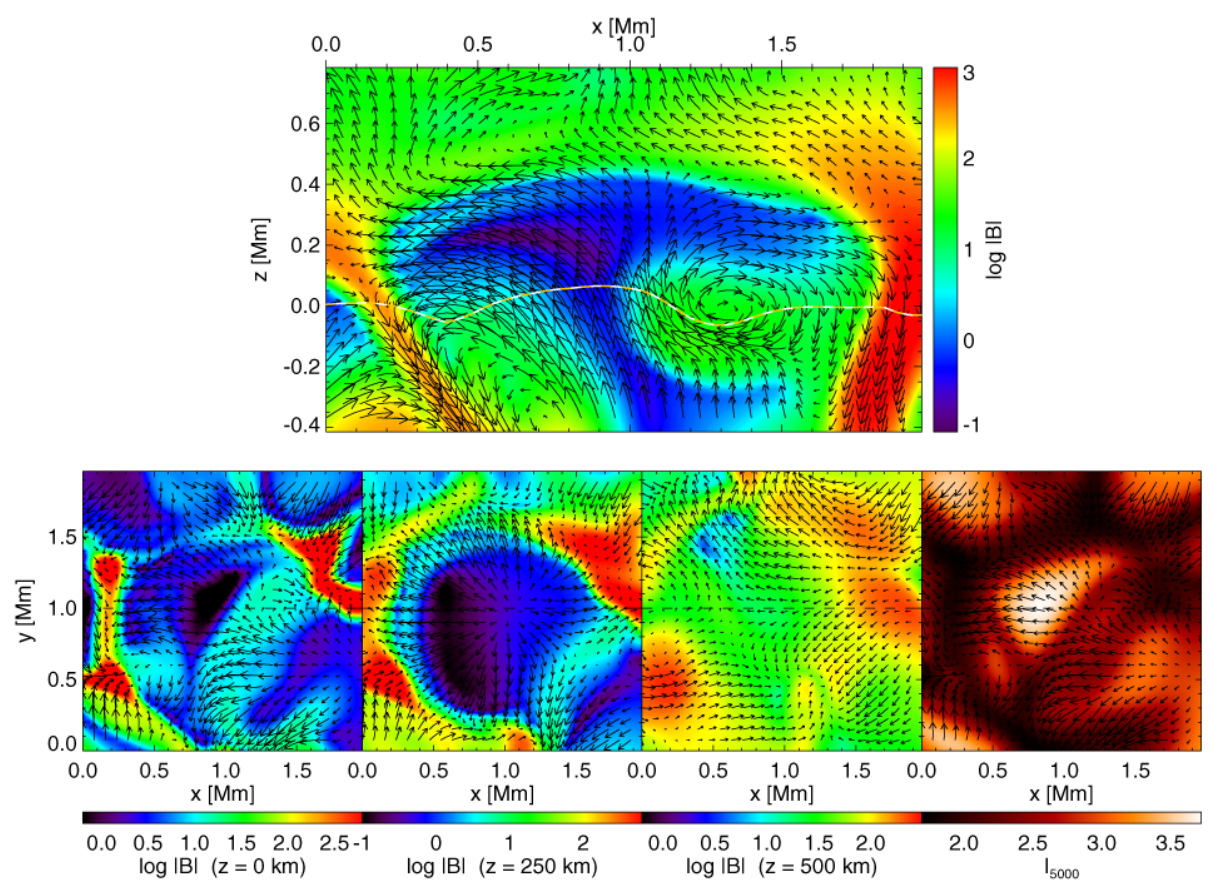

Fig. 13 Flux expulsion in a close-up from a MHD simulations by Schaffenberger et al. (2005): Logarithmic magnetic field strength in a vertical cross-section (top) and in three horizontal cross-sections (bottom) at heights of $0 \mathrm{~km}, 250 \mathrm{~km}$, and $500 \mathrm{~km}$. The emergent intensity is displayed in the rightmost panel. The arrows represent the velocity field in the shown projection planes. The white line in the upper panel marks the height of optical depth unity

seen in the simulations. It is observed that the horizontal field component in the granular interiors is stronger than the vertical component (Lites et al. 1996, 2007, 2008; Orozco Suárez et al. 2007). HIFs are also clearly present in simulations (Schaffenberger et al. 2006; Schüssler and Vögler 2008; Steiner et al. 2008) and are in good agreement with the observations.

The direction of the horizontal magnetic field, which is continuously lifted to the upper photosphere and lower chromosphere, varies. Consequently, current sheets form where different field directions come close to each other. In the simulations by Schaffenberger et al. (2006), a complex stacked meshwork of current sheets is generated at heights from $\sim 400 \mathrm{~km}$ to $\sim 900 \mathrm{~km}$. The lower limit of this range, which is the typical height of the small-scale canopies can be considered as the upper boundary of the photosphere.

\subsection{Internetwork Chromosphere}

In recent years models have been extended in height to include the chromosphere. Modeling this layer is an intricate problem as many simplifying assumptions, which work fine for the lower layers, are not valid for the thinner chromosphere. Rather, time-dependent three-dimensional non-equilibrium modeling is mandatory. This is in particular true for the radiative transfer, for which deviations from the (local) thermodynamic equilibrium should be taken into account. Numerically, this is a demanding task. It is unavoidable to 
make simplifications and compromises when implementing at least the most important nonequilibrium effects in a time-dependent multi-dimensional simulation code. A practicable way is to start with simplified models and increase the amount and the accuracy of physical ingredients step by step. In their pioneering work, Carlsson and Stein (1994, 1995) implemented a detailed radiative transfer, which was affordable by restricting the simulation to one spatial dimension. This simplification made it necessary to implement an artificial piston below the photosphere to excite waves as the convection cannot be realistically simulated in one spatial dimension. The high computational costs for such detailed radiative transfer calculations forced Skartlien et al. (2000) to use a simplified description for their three-dimensional model. Nevertheless, their treatment included scattering. Simplifications of the radiative transfer are necessary for three-dimensional simulations in order to make them computationally feasible. This class of $2 \mathrm{D} / 3 \mathrm{D}$ numerical simulations cover a small part of the near-surface layers and extent vertically from the upper convection zone to the middle chromosphere. This way the shock-waves are excited by the simulated convection without any need for an artificial driver. The chromospheric layer of these models is usually characterized by intense shock wave action, putting high demands on the stability of numerical codes. Wedemeyer et al. (2004) made experiments with simplified 3D models without magnetic fields, using $\mathrm{CO}^{5}$ BOLD (Freytag et al. 2002). As in the aforementioned simulations, they found that overshooting convection in the photosphere triggers acoustic waves that propagate upwards and steepen into shock fronts. The result is a dynamic layer above a height of $\sim 700 \mathrm{~km}$, which is composed of hot shock fronts and cool post-shock regions. The gas temperature in horizontal cross-sections through the model exhibits highly dynamic mesh-like pattern with spatial scales comparable to the granulation. The same can be seen in the follow-up simulations by Schaffenberger et al. (2005), which include weak magnetic fields (see Fig. 14). The gas temperature in the $\mathrm{CO}^{5} \mathrm{BOLD}$ model chromospheres range from about $7000 \mathrm{~K}$ down to $2000 \mathrm{~K}$, owing to the adiabatic expansion of the postshock regions. A similar pattern is also present in the simulations by Martínez-Sykora et al. (2008). The temperature range is very similar in both models, but the temperature amplitudes differ. Some snapshots of the simulation by Martínez-Sykora et al. (2008) also show a double-peaked temperature distribution at chromospheric heights, but the cool background component is usually much weaker than in the $\mathrm{CO}^{5} \mathrm{BOLD}$ model. Possible reasons for the differences are related to the numerical treatment of the radiative transfer in the upper layers. A shock-induced pattern can already be perceived in the temperature maps by Skartlien et al. (2000), although it less pronounced due to the relatively coarse grid spacing in this earlier simulation.

Not only the modeling but also the observation of the shock-dominated layer (hereafter referred to as "fluctosphere", see Sect. 5) is non-trivial. A clear detection in Ca II H, K or the infrared lines requires a high spatial, temporal, and spectral resolution, all at the same time. A too broad filter wavelength range leads to significant contributions from the photosphere below. The fluctospheric pattern is then easily masked by a reversed granulation signal. The situation is complicated by the fact that both patterns have very similar spatial scales, i.e. roughly granulation scales. This is due to the fact that the generation of both patterns is due to processes in the low photosphere. This is illustrated in Fig. 15, which shows preliminary synthetic intensity maps in the Ca II infrared line at $\lambda=854 \mathrm{~nm}$. The maps were calculated with the non-LTE radiative transfer code MULTI (Carlsson 1986) column by column from the model by Leenaarts and Wedemeyer-Böhm (2006). We use the non-equilibrium electron densities, which are output from the time-dependent simulation. The top rightmost panel of Fig. 15 shows the mesh-like pattern in the line core, whereas the reversed granulation is visible in the line wing (middle column). Even further out in the wing, the granulation 

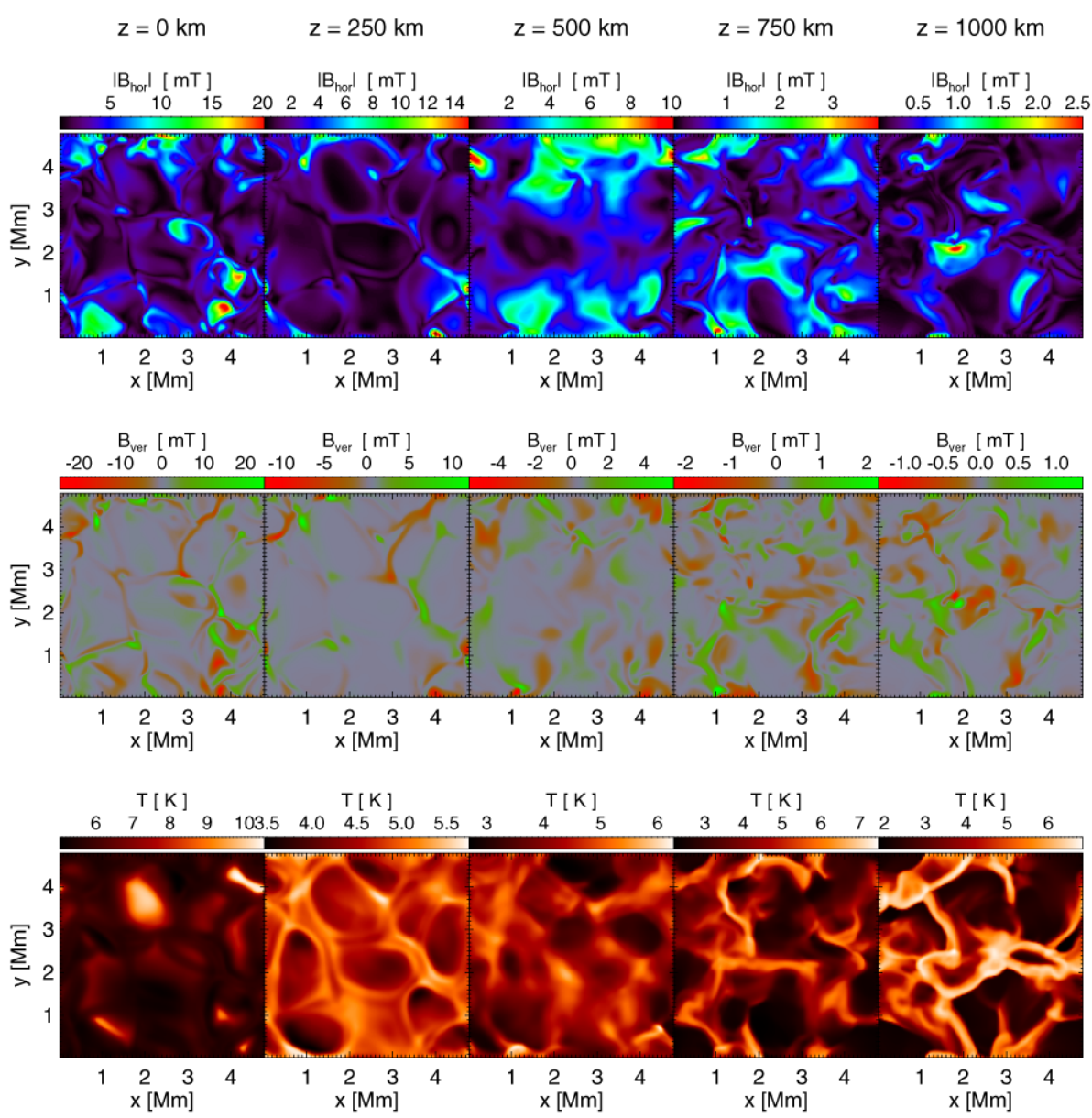

Fig. 14 Horizontal cross-sections through the model by Schaffenberger et al. $(2005,2006)$ showing the horizontal magnetic field component (top), the vertical component (middle row), and the gas temperature (bottom) at different heights: $z=0 \mathrm{~km}$ (granulation), $250 \mathrm{~km}$ (reversed granulation), $500 \mathrm{~km}, 750 \mathrm{~km}$ (fluctosphere), and $1000 \mathrm{~km}$ (from left to right)

pattern appears (left column). The mesh-like fluctosphere pattern can be seen $\mathrm{Ca} \mathrm{H}, \mathrm{K}$, and the IR triplet, too.

A comparison of the line core map with the temperature maps in Fig. 14 shows that primarily the hottest regions of the pattern are seen in the $\mathrm{Ca}$ intensity, whereas a lot of atmospheric fine-structure remains invisible. The hot regions are caused by "collision" of neighboring shock fronts, ultimately compressing the gas in the region in-between and rising its temperature. This effect enhances in particular the $\mathrm{Ca}$ brightness at the vertices of the mesh. These small bright areas most likely are observed as $\mathrm{Ca}$ grains, while the emission along the mesh is so faint that it is hard to detect. The formation of Ca II grains by propagating shock waves was already explained by Carlsson and Stein (1997) over a decade ago. The fact that their detailed 1D simulations closely match observations of grains, clearly shows that $\mathrm{Ca}$ II grains are indeed a phenomenon related to shock waves. In 1D but also in $3 \mathrm{D}$, the formation takes place at heights of $\sim 1 \mathrm{Mm}$ above optical depth unity. In both 

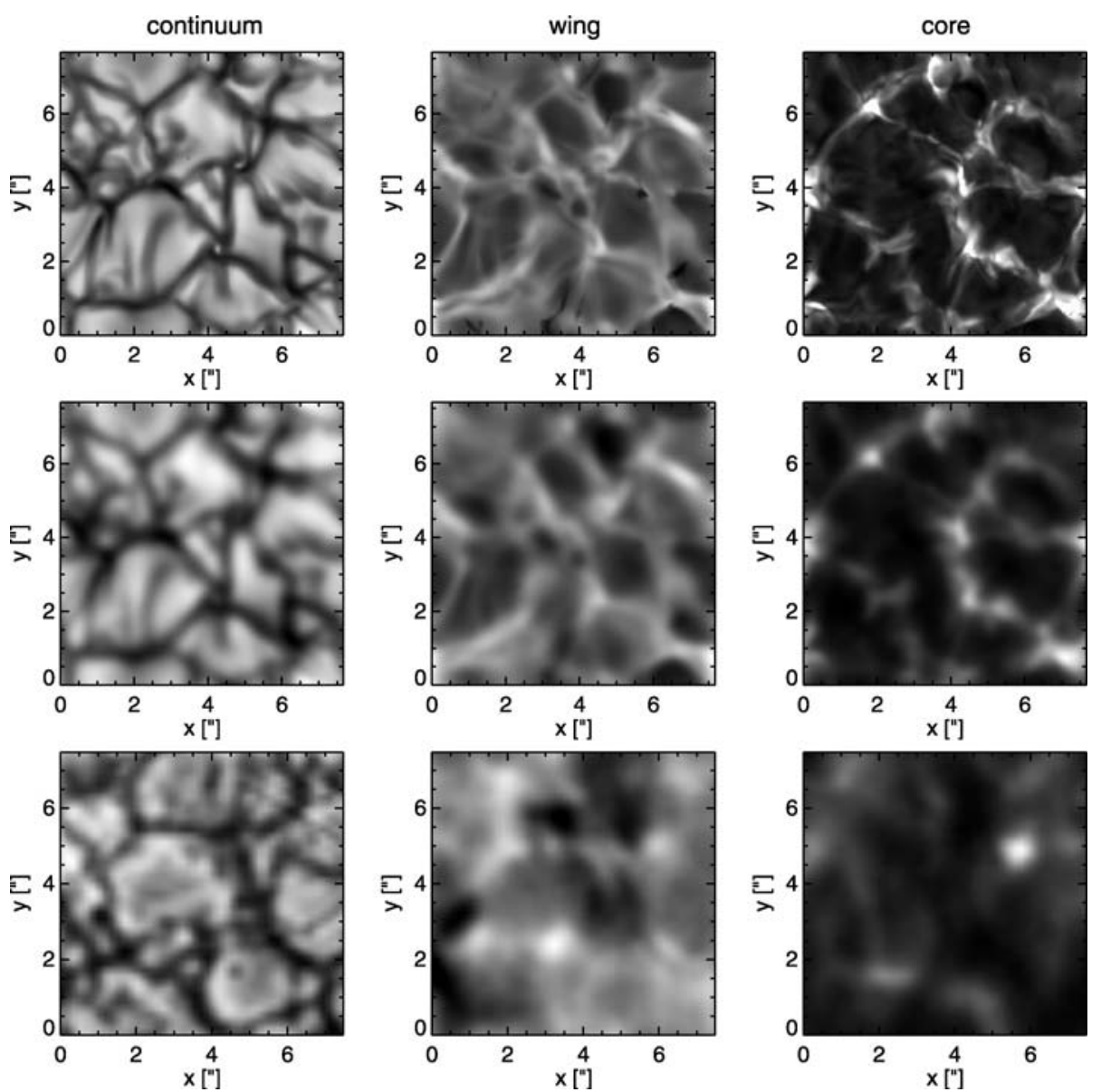

Fig. 15 Small-scale structure of the solar atmosphere seen in the Ca II infrared line at $854 \mathrm{~nm}$ continuum (left column), line wing (middle), and line core (right column). Top row: Synthetic maps based on a simulation with non-equilibrium hydrogen ionization; middle row: after application of a PSF and filter transmission; bottom: observations with IBIS at the DST (Courtesy of F. Wöger). See text for details

cases, the shocks propagate upwards into down-flowing material. The difference, however, is that in 1D shocks are plane-parallel so that interaction between individual waves is essentially reduced to shock-merging and shock-overtaking. In 3D, shock wave interaction is more complex. And still, the compression zones between shocks - the most likely candidate for grain formation in 3D-moves upwards with the waves and thus certainly show very similar observational signatures. While it seems to be well established that Ca grains are produced by shock waves, some details of the formation process have to be revisited in a $3 \mathrm{D}$ context.

However, the grains might just be the "tip of the iceberg". Progress in observational techniques and instrumentation now finally allow us to detect the dark details of the fluctosphere. The middle row of Fig. 15 illustrates this observational effect. A point spread function (PSF) has been applied to the synthetic maps. The PSF accounts for a circular, unobstructed aperture of $70 \mathrm{~cm}$ diameter and a non-ideal Voigt-like contribution due to instrumental stray-light and atmospheric seeing. Finally, the degraded maps are integrated 
over wavelength with a synthetic transmission filter with a FWHM of $5 \mathrm{pm}$. The assumptions are rather optimistic and represent excellent observational conditions. And yet the resulting image degradation has a significant effect on the visible patterns. Obviously, a lower spatial or spectral resolution would further suppress the faint mesh-like pattern in the line core. Please note that the calculations are still preliminary. A full 3D treatment of the radiative transfer and the included scattering, which will soon be possible, might increase the area of enhanced brightness. Also it is not clear yet how the possible interaction of the shock waves with the overlying "canopy" field would alter the properties of the pattern and its observational mesh/grain signature. The resulting pattern nevertheless in many aspects resembles the recent observations by F. Wöger et al. with (i) the Interferometric BIdimensional Spectrometer (Cauzzi et al. 2008, IBIS) at the Dunn Solar Telescope (DST) of the National Solar Observatory at Sacramento Peak (Wedemeyer-Böhm and Wöger 2008) and (ii) with the German Vacuum Tower Telescope (VTT) at the Observatorio del Teide (Wöger et al. 2006). See the lower row of Fig. 15 for examples of IBIS data.

Based on the models by Wedemeyer et al. (2004), weak magnetic fields were taken into account in the simulations by Schaffenberger et al. $(2005,2006)$ and Steiner et al. (2008) (see Fig. 14). Different initial magnetic field configurations and strengths from $B_{0}=10 \mathrm{G}$ to $20 \mathrm{G}$ were tried, all resembling quiet Sun internetwork conditions (see Wedemeyer-Böhm et al. $2005 \mathrm{~b}$ for an experiment with $B_{0}=100 \mathrm{G}$ ). The computational domains again comprise several granules and extend into the chromosphere, typically to heights of $\sim 1400 \mathrm{~km}$. The MHD models are very similar to their hydrodynamic precursors with respect to structure and dynamics. The ubiquitous shock waves produce a very similar pattern in the gas temperature but also shape the small-scale structure of the magnetic field in the upper model atmosphere. Consequently, the magnetic field in the fluctosphere is highly dynamic and has a complex topology. A look at horizontal cross-sections at different heights in Fig. 14 implies that the field in the upper layers is much weaker $(|B|<50 \mathrm{G})$ and more homogenous than in the photosphere below. On the other hand, the fluctospheric field evolves much faster. The horizontal field component $B_{\text {hor }}$ in the range $500 \mathrm{~km}$ to $750 \mathrm{~km}$ is (i) stronger than the vertical one, $B_{\mathrm{z}}$ and (ii) has a rather large filling factor there.

In the small-scale internetwork simulations carried out with $\mathrm{CO}^{5} \mathrm{BOLD}$, the strongly varying surface of plasma $\beta=1$ is found on average at heights of the order of $1000 \mathrm{~km}$ to $1400 \mathrm{~km}$ or even higher, depending on model details. Heights of the same order are also found by, e.g., Hansteen (2007). The exact location certainly depends on the field strengths in the internetwork, which are still under debate. Instead of plasma $\beta=1$, one can also talk about an equivalent surface, where sound speed and Alfvén speed are equal. It makes clear that these regions are important for the propagation and eventual dissipation. Simulations show that this surface indeed separates two domains that differ in their dynamical behavior: A slow evolving lower part and a highly dynamic upper part. This is certainly related to the finding that wave mode conversion and refraction occurs under the condition of plasma $\beta \approx 1$ (Rosenthal et al. 2002; Bogdan et al. 2003; Cally 2007; Steiner et al. 2007). The current sheets, which are present below and above the plasma $\beta=1$ surface, differ in their orientation. While they are mostly stacked with horizontal orientation in the lower part down to the top of the small-scale canopies at the boundary to the photosphere, the thin current sheets above plasma $\beta=1$ are formed along shock fronts and can thus show oblique or even vertical orientation. 


\subsection{Large-Scale Simulations}

The models described in Sects. 4.1 and 4.2 do not take into account the large-scale canopy fields but rather concentrate on the small spatial scales of quiet Sun internetwork regions. In contrast, the simulations discussed in this section comprise larger computational domains. To make this possible, one usually has to make compromises such as, e.g., reduce the spatial resolution or develop efficient numerical methods. Stein et al. (2006) made impressive progress by extending the computational box towards supergranulation scales. Their models do not include the upper atmosphere but extend deep into the convection zone. Gudiksen and Nordlund (2002, 2005a), on the other hand, succeeded in creating time-dependent numerical models, which extend from the photosphere all the way into the corona. An important aspect, which can be investigated with this kind of models, it the (magnetic) connection between the atmospheric layers all the way from the top of the convection to the corona (see also Abbett 2007). Furthermore, extended simulations allow for investigating phenomena that are connected to spatial scales between granulation and supergranulation. For instance, the simulations by Hansteen and Gudiksen (2005) and Hansteen et al. (2006) revealed the formation of dynamic chromospheric features similar to dynamic fibrils. Being driven by upward propagating waves in the chromosphere, they are an example of the coupling between different atmospheric layers. Another type of coupling is provided in the form of horizontal magnetic flux structures with extensions of a few Mm, which emerge from the upper convection and rise upwards through the atmosphere. See Cheung et al. (2007) and Martínez-Sykora et al. (2008) for recent examples of flux emergence simulations. The simulations by Leenaarts et al. (2007) confirm once more (cf. Carlsson and Stein 2002) that the ionization degree of hydrogen has to be treated in non-equilibrium in the upper atmosphere. Although the simulation is two-dimensional, it features weak-field sub-canopy domains with upward propagating shock waves and a magnetic-field dominated "canopy" domain above (see their Fig. 1). A strong coupling of the individual layers is very obvious.

\section{An Updated Picture of the Quiet Sun Atmosphere}

The results of the previous sections are summarized in a schematic sketch of the quiet Sun atmosphere (see Fig. 16) with particular emphasis on the low atmosphere in internetwork regions. It is based on (and should be interpreted in comparison with) recent sketches by, e.g., Judge (2006), and Rutten (2006, 2007) but contains many modifications to incorporate new results derived from observations and numerical simulations.

\subsection{The Large-Scale Magnetic Field}

The large-scale building blocks of the quiet Sun atmosphere are the magnetic network patches, which outline supergranulation cells. The large-scale convective flows (see long arrows) advect magnetic field to the lanes of the supergranulation. Consequently, the magnetic field is highly structured and concentrated close to the "surface" $\left(\tau_{500}=1\right)$ with kG field strengths. The visible result is the so-called magnetic network (see Fig. 7. More recent observations with high spatial resolution (e.g., Orozco Suárez et al. 2007) reveal that the magnetic network patches consist of a conglomerate of smaller magnetic elements or "flux bundles" of different field strength with a wealth of substructure. This finding is incorporated in Fig. 16, in contrast to earlier sketches that feature the magnetic network as rather massive flux tubes. The heights where sound speed and Alfvén are equal $\left(c_{\mathrm{s}}=c_{\mathrm{A}}\right)$, or equivalently where the 


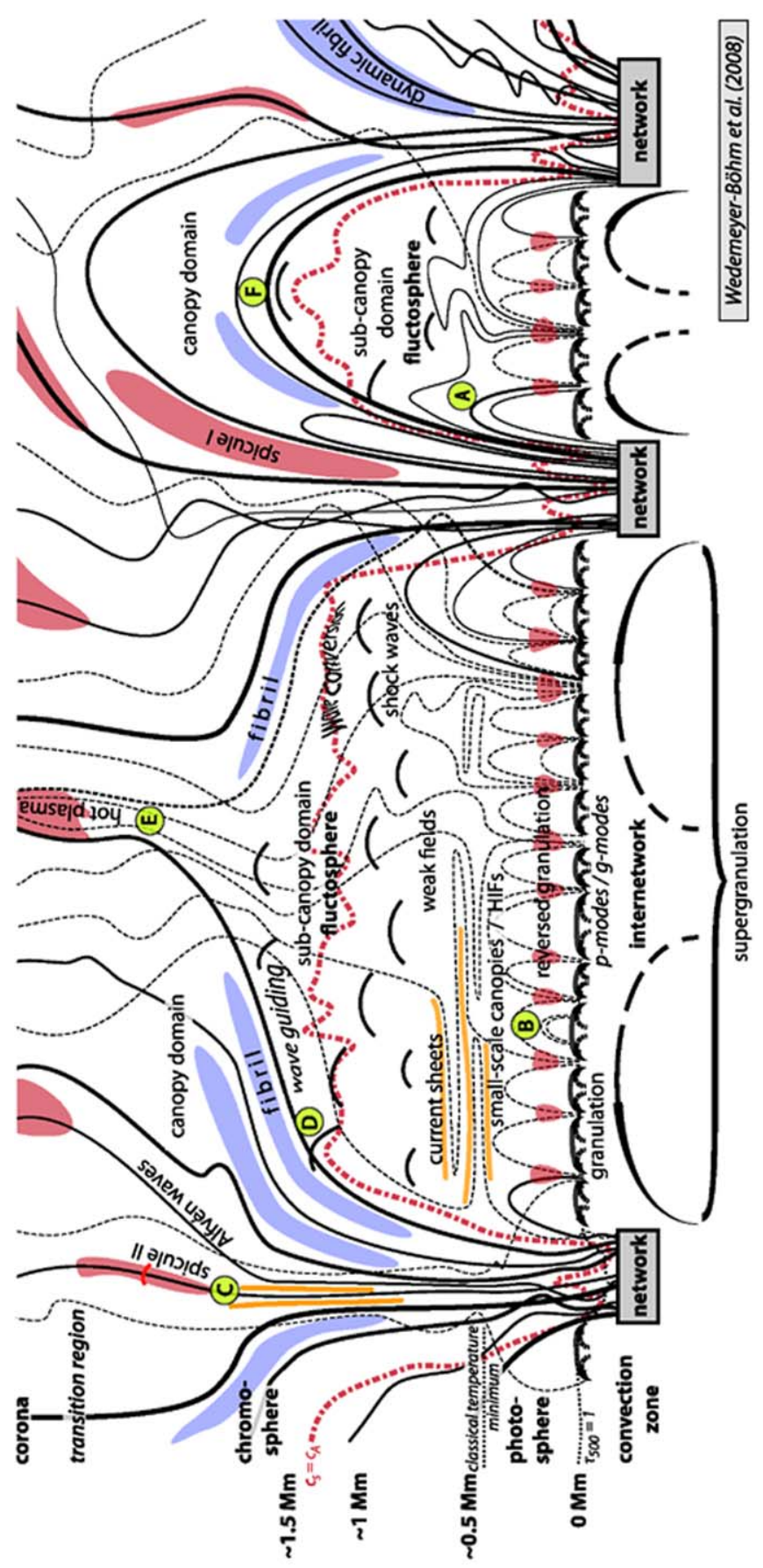

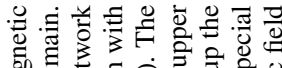

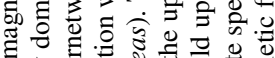

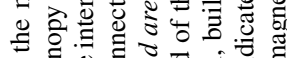

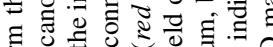
음.

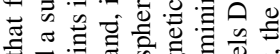
讨

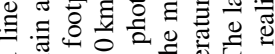

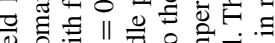

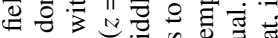

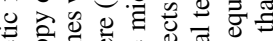

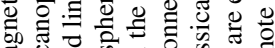
药 요 ôि

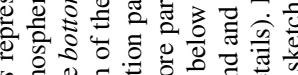
记

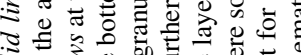

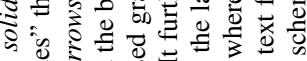
o 5 的

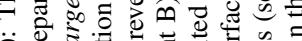
o

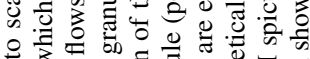

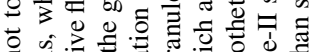

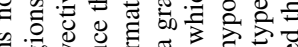

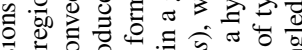
क

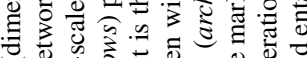

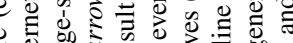

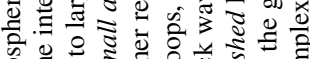

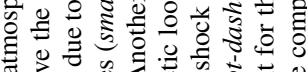
क

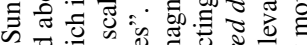

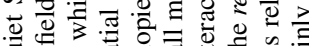

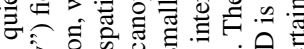

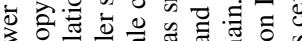
들

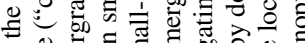

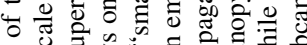
0 क

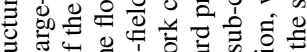

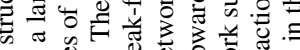

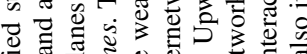

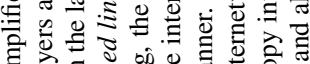

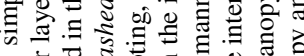
บ

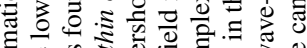
의

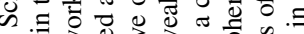

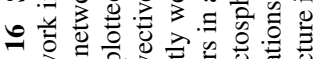

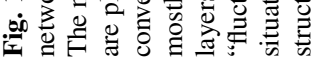


plasma $\beta=1$, will certainly show large variations, depending on the (local) field strength. It may even reach below the surface of optical depth unity (at a reference wavelength of $500 \mathrm{~nm}$ ) within strong field concentrations but may stay up at a few hundreds kilometers in weaker network patches. And still the variation in field strength and topology, incl. the width of the network patches, is even larger than can be presented in the simplified sketch here.

The magnetic field spreads out in the layers above the patches. Depending on the polarity of neighboring flux concentrations, they can form funnels or connect via loops that span the internetwork regions in-between. These two cases are illustrated in Fig. 16 in a simplified way. In the classical picture, the large-scale field enclosing the weak-field internetwork regions is referred to as "magnetic canopy". The corresponding flux funnels are often depicted with a wineglass-like geometry and have their footpoints in the photospheric network only. In reality, where the third spatial dimension offers an important additional degree of freedom, the field topology is more complex (see, e.g., Gudiksen 2006; Peter et al. 2006; Jendersie and Peter 2006; Schrijver and van Ballegooijen 2005). Schrijver and Title (2003) state that as much as half of the field could actually be "rooted" in the internetwork regions. From there, it can connect directly to the coronal field or via small loops to the photospheric network. The network patches could thus be surrounded by "collars" of loops with spatial scales comparable to one or a few granules. Consequently, the concept of a regular canopy structure seems questionable. Instead, the field topology should rather be understood as a set of individual field lines. Nevertheless, we stick here to the term "canopy" but use it in a wider sense. The height of the canopy and the field structure as a whole varies significantly from region to region and with time. The height indicators to the left in Fig. 16 should therefore only be used for rough orientation. In principle, the lower boundary of the "canopy" field separates two distinct domains: a canopy domain and a subcanopy domain. In reality, however, the boundary is certainly less strict than the sketch may imply. Rather, the magnetic field of both domains may be interconnected, e.g., by small loops, which extend on granular scales (point A). This way, the dynamics of the internetwork photosphere could have a direct influence on the properties of the upper layers, e.g., with respect to wave propagation and heating.

\subsection{The Canopy Domain}

The canopy domain is dominated by (large-scale) magnetic fields. It is this layer, which, due to the emission in $\mathrm{H} \alpha$, appears as a purple-red rim at the beginning and end of a total solar eclipse. Therefore, only the canopy domain represents the chromosphere in a strict and original sense. At a closer look, a rich fibrilar structure can be seen in chromospheric $\mathrm{H} \alpha$ observations. They are found in rosette-like formations that funnel out from the magnetic network below and in many cases connect to neighboring network fields. A few fibrils are shown in Fig. 16 in connection with plasma that is trapped in the chromospheric field. Such fibrils and also the larger dynamic fibrils (Hansteen et al. 2006; Langangen et al. 2008a, 2008c shown at the right in the figure here) are an integral part of the quiet Sun chromosphere and even more frequent than can be shown in the 2D sketch here. According to De Pontieu et al. (2007c), fibrils could be the result of chromospheric shock waves that occur when convective flows and global oscillations leak into the chromosphere along the field lines of magnetic flux concentrations. In general, magnetohydrodynamic waves are an integral and ubiquitous part of the canopy domain. (Alfvén waves are indicated in Fig. 16 but represent just one of several possible wave modes.) Such perturbations can be excited by a number of processes, e.g., by the shuffling and braiding of the magnetic footpoints in the photosphere by convective flows. As the large-scale magnetic field continues from the 
lower layers into the transition region into the corona above, the whole canopy domain is dynamically coupled. Again, it must be emphasized that the field topology is certainly more complex than can be expressed in the sketch here (see, e.g., Fig. 7). Indeed, Schrijver and van Ballegooijen (2005) state that instead of the plasma- $\beta$ surface being closely connected to the (classical) canopy, regions with low and high $\beta$ can well be mixed up into the corona.

As already mentioned in Sect. 3, a most obvious constituent of the chromosphere, at least when observed at the solar limb, are spicules (see, e.g., De Pontieu et al. 2004). Now, two types of spicules are distinguished based on differences in their dynamic behavior (De Pontieu et al. 2007a). Spicules of type I are the result of shock waves that are excited by disturbances in the photosphere (e.g., in connection with p-modes) and propagate from there along the magnetic field lines photosphere into the upper layers (Hansteen et al. 2006; Rouppe van der Voort et al. 2007). Spicules of type II, on the other hand, are more dynamic but thinner, exhibit higher velocities and have shorter lifetimes (see, e.g., Langangen et al. 2008b). They are most likely generated by magnetic reconnection events. Alfvén waves, which by many are considered as an ubiquitous phenomenon in the upper atmosphere, can be detected in connection with spicules (De Pontieu et al. 2007b). An example is drawn in the upper chromosphere above some vertically orientated current sheets (point $\mathrm{C}$ ).

Another ingredient of the sketch are blobs of hot plasma in the corona, although their exact position and shape needs further investigation. De Pontieu et al. (2003) showed that the emission is not correlated with the centers of flux concentrations. Rather, the emission seems to appear at random locations. Although De Pontieu et al. (2003) refer to "moss" (Berger et al. 1999; Fletcher and De Pontieu 1999), which is related to active regions, there is no obvious reason why the situation should be different for the quiet Sun corona. Also, hot plasma regions like the one marked with "E" in the sketch are certainly not preferentially located directly above the middle of an internetwork region. In reality, the entangled and skewed field topology will make such blobs-if existent in the way depicted here-appear rather uncorrelated with the field topology of the underlying magnetic network.

\subsection{The Sub-Canopy Domain}

The magnetic field in the sub-canopy domain is mostly weak (see, e.g., Trujillo Bueno et al. 2004; Orozco Suárez et al. 2007), so that the plasma is larger than one in the lower layers. There, the field is essentially passively advected by the hydrodynamic flow fields. Convective motions and overshooting at the "surface" are the fundamental structuring agents, making the granulation the dominant spatial scale. Nevertheless, the weak fields in the subcanopy domain most likely connect at least partially with the stronger canopy field. This feature is taken into account as integral part of the atmosphere sketch. Unfortunately, the presentation remains rather speculative at this point as many details of how and where the connections exactly take place are still unknown.

Beside the magnetic field, the consequences of convective overshooting allow to divide the subcanopy domain into layers with distinct dynamics (from bottom to top): low photosphere, middle photosphere, high photosphere, fluctosphere.

The Lower and Middle Photosphere exhibit the visible imprints of the solar surface convection. The granulation in the low photosphere is directly produced by small-scale convection cells (see, e.g., Nordlund and Dravins 1990), while the reversed granulation in the middle photosphere is a second-order effect. Gas is brought up by convective overshooting in the granule interiors, adiabatically expanding and cooling. It streams down again in the intergranular lanes, where it is compressed and heated. In addition, p-modes, i.e. global oscillations, and local acoustic events are important ingredients of the photospheric dynamics. 
Recently, Straus et al. (2008) presented new support for the idea that gravity waves could play an important role, too.

The usually weak magnetic field is brought up from the convection zone below and/or possibly locally generated by small-scale dynamo action close to the surface. In the photosphere, the weak field is more or less passively advected towards the intergranular lanes but also towards the upper photosphere. The resulting field concentrations in the lanes become visible as very small and confined structures, e.g., in G-band images (see, e.g., de Wijn et al. 2008, for a recent example). In general, the internetwork field in the photosphere exhibits significant inclination and mixed polarity (see, e.g., Martínez González et al. 2008; Orozco Suárez et al. 2007). The granule interiors may become virtually field-free if there is no supply of magnetic fields with the warm convective upflows. Such voids are enclosed by small-scale canopies. Over most of the granulation, the horizontal field component is stronger than the vertical. This effect is observed as "horizontal internetwork fields" (HIFs). Magnetic field can emerge also in the form of small loops, which may have footpoints even within a granule (see point B in Fig. 16). This process, which was observed by Centeno et al. (2007), most likely adds to the accumulation of field above granules. In addition to emerging loops, Stein and Nordlund (2006) report on flux that is submerging and thus disappears from the surface.

The Upper Photosphere marks the boundary between the photosphere, which is controlled by the effects related to convective overshooting, and the wave-dominated layer above. This boundary can roughly be placed at the height of the classical temperature minimum. There, the temperature structure appears smoothed out and less structured than above and below; it is here that the average temperature amplitudes are smallest. It is roughly the height where the UV continuum at $160 \mathrm{~nm}$ is formed (cf. TRACE passbands). The upper photosphere is the layer, where the small-scale canopies have their top and where stacked (horizontal) current sheet become most obvious. This layer can be seen as a kind of (dynamical) insulation between the internetwork photosphere and fluctosphere. This effect becomes obvious in simulations when starting from an initial condition which feeds in field at the lower domain boundary. The photospheric field is built-up rather quickly but the field above only after a time delay because it only slowly spreads into the strongly subadiabatic stratification of the upper photosphere.

The Fluctosphere The shock-dominated domain in subcanopy internetwork regions (see Sect. 4.2), is referred to as "fluctosphere" by Wedemeyer-Böhm and Wöger (2008), while Rutten (2007) uses the term "clapotisphere". It is located between the photosphere and the part of the chromosphere visible in $\mathrm{H} \alpha$. It is composed of propagating and interacting shock waves (with weak field only) and intermediate cool post-shock regions. Ideally, the wave fronts would expand spherically, while moving in vertical direction. In reality, they are deformed by running into an inhomogeneous medium of downflowing gas, which was shaped by precursory wave trains. The horizontal expansion of the fronts inevitably causes interaction between them. A visible result is the formation of $\mathrm{Ca}$ grains at heights, which traditionally would be assigned to the low chromosphere. The waves are excited in the photosphere below via different processes, which are related to convection (e.g., exploding granules), overshooting, and p-modes. The magnetic field in the fluctosphere is rather weak and is therefore mostly passively shuffled around by the shock waves. The result is a very dynamic and entangled field. The strongly varying surface of plasma $\beta=1$ or in this context better $c_{\mathrm{s}}=c_{\mathrm{A}}$ is most likely located at heights of the order $1000 \mathrm{~km}$ to $1500 \mathrm{~km}$ or even higher (see Sect. 4.2). There, the conditions allow wave mode conversion, so the parts of the fluctosphere 
below and above can show a somewhat different dynamical behavior. In the upper part, the weak fields become more important and rapidly moving filaments of enhanced field strength are generated. The propagating shock waves nevertheless remain the dominating structuring agent. A consequence, however, is that the current sheets are only stacked at plasma $\beta>1$. Above, they are less regular as they are formed in the narrow collision zones of shocks, where the magnetic field is occasionally compressed. This shock-induced magnetic field compression might qualify as a (minor) heating process with potential consequences for the chromospheric energy balance.

The fluctosphere is not directly visible in $\mathrm{H} \alpha$ (in the line core at least) and is thus not a part of the chromosphere in a strict sense. It seems advisable to reserve the term chromosphere for the fibrilar canopy domain as visible in $\mathrm{H} \alpha$ (or in the very line cores of the Ca II lines). However, the fluctospheric shock waves could still leave an imprint in chromospheric diagnostics by interacting and penetrating the canopy field. On the other hand, the fluctosphere is also no part of the photosphere, although causally connected via the shock waves that propagate upwards from the low photosphere. The fluctosphere could be regarded as a second-order effect only, in contrast to the granulation and reversed granulation, which are direct consequences of the solar surface convection.

\subsection{Shock Waves Meet the "Canopy"}

Some details of Fig. 16 concern the interplay of propagating waves and the magnetic canopy. There are certain zones in these magnetic structures that act as mode conversion zone (Bogdan et al. 2003; Cally 2007), e.g., converting incoming acoustic waves into other modes, such as fast and slow magnetoacoustic waves. It is thus possible that such converted waves continue to propagate along the canopy field lines as some kind of "canopy waves". For simplicity, such a zone is marked by "wave conversion" at the "outer" boundary between canopy and subcanopy domain in the figure. Generally, such zones can be located everywhere in the structure where sound speed and Alfvén speed are of equal magnitude.

Furthermore, refraction and even reflection of waves can occur in such zones. As for the mode conversion, details depend on the relative orientation of the incoming wave and the magnetic field (Hasan et al. 2008). A wave can remain barely affected by the field when traveling perpendicular to the field lines, e.g., upwards in a vertical flux concentration. On the other hand, significant (relative) inclination can even result in total internal reflection for some wave modes (Rosenthal et al. 2002). In general, it can be assumed that the (acoustic) shock waves coming from the fluctosphere are guided by the magnetic canopy (e.g., point D in Fig. 16). Consequently, waves might follow the canopy field upwards and compress and heat the gas trapped between chromospheric "funnels" (point E). In closed loop regions, strong waves could push into the canopy from below and compress the magnetic field (location F). Depending on the local field configuration and the properties of the incoming wave, such an event could eventually trigger reconnection events. It could contribute to chromospheric heating. It certainly would not be only limited to the locations indicated in the sketch but occur more often in complex 3D field configurations. On the other hand, a regular closed structure as in the figure could possibly refract the waves from below such that they are "focussed" in the top of the subcanopy domain, amplifying their effect on the canopy field. A possible-although speculative-result could be the triggering of "nanoflares", although they are initiated by other mechanisms at other locations, too. That the upwards propagating waves interact with the canopy field is implied by observations in $\mathrm{H} \alpha$. The dynamic behavior of the chromospheric fibrils is reminiscent of strings that sway back and forth in reaction to the quasi-continuous impact of 
waves from below (point F). Under certain conditions, the shock waves might actively deform the field configuration of the magnetic canopy. Magnetoacoustic waves can already enter network flux concentrations in the photosphere, where the inclined magnetic field lines act as "magnetoacoustic portals" (Jefferies et al. 2006). The observations of socalled "acoustic shadows" provide observational evidence for the interaction of acoustic waves with the field around network footpoints (Krijger et al. 2001; McIntosh et al. 2001; McIntosh and Judge 2001).

\subsection{Probing the Upper Atmosphere}

With the currently available diagnostics for the chromosphere, observations of the subcanopy domains are problematic. The $\mathrm{H} \alpha$ line core samples only the "canopy domain", whereas observations in the line wing reveal a background that most likely is dominated by the reversed granulation at much lower heights. It seems questionable if the layer inbetween - the fluctosphere-can be observed in the $\mathrm{H} \alpha$ line wing at all in internetwork regions. Polarimetric measurements in the He $10830 \AA$ line (see Sect. 3) principally allow for the determination of the magnetic structure in a slab located between $1000 \mathrm{~km}$ and $2000 \mathrm{~km}$. The formation of this line requires coronal illumination in the UV, resulting in complete absence of any photospheric contamination. However, the main contribution in the He $10830 \AA$ line comes from layers slightly above the fluctosphere. The $\mathrm{Ca} \mathrm{H} \& \mathrm{~K}$ and IR lines in principle would allow observations of the fluctosphere if very narrow filters are used. Otherwise, the detected intensity is "contaminated" with radiation from layers below. Very often, Ca observations with too broad filter prominently show the reversed granulation (see Figs. 7d and 11), which is easily mistaken as chromospheric signal. Very narrow filters, on the other hand, make it necessary to properly correct for Doppler shifts. A solution is fast scans through the Ca II IR lines with new imaging polarimeters such as IBIS (Cauzzi et al. 2008; Kleint et al. 2008) or CRISP (Scharmer et al. 2008), or spectro-polarimeters like SPINOR (Socas-Navarro et al. 2006). The extended formation height ranges and the non-equilibrium conditions, under which the inner parts of these lines are formed, complicate the interpretation and the derivation of the atmospheric structure. A promising alternative are the (sub-)millimeter continua, which will become accessible with the Atacama Large Millimeter Array (ALMA) a few years from now. Although technical details of this new type of observation render the construction of brightness temperature maps a certainly very complicated task, the scientific results could significantly contribute to our understanding of the solar atmosphere at chromospheric heights (Loukitcheva et al. 2008; Wedemeyer-Böhm et al. 2007).

\section{Conclusions}

The solar atmosphere is a very dynamic and inhomogeneous multi-scale system. Its individual components are coupled; some of them even show a kind of hierarchical self-similarity. Examples are the observational imprints of sub-surface convection, with a continuous spectrum of scales from below granulation scales to above supergranulation scales, and magnetic fields, which also exhibit similar features over a large range of spatial scales.

Despite great progress on the theoretical and observational sides, which go hand in hand, we are still missing an ultimate, comprehensive picture of the quiet Sun atmosphere. But at least we can now see what is needed for a corresponding numerical simulation. First, the computational domain should be large enough to encompass a few supergranulation cells 
while the spatial resolution must still be high enough to capture important processes that occur on scales smaller than granulation. The vertical couplings make it necessary to consider an extensive height range. The corona and chromosphere can only be treated realistically when including the important driving motions in the layers below, i.e. in the photosphere and (at least) the upper part of the convection zone. While many simplifying assumptions can be made for the lower parts of such a model, the layers above the (middle) photosphere require a numerically complicated and thus computationally expensive non-equilibrium modeling approach, e.g., a realistic treatment of hydrogen ionization etc. The production of such a comprehensive model — and analogous models for, e.g., active regions-is thus very involved and can be regarded as one of the current challenges in (computational) solar physics.

On the observational side, we must continue to push forward the instrumental possibilities towards higher resolution in the spatial, temporal, and spectral domains-all at the same time. In addition, the (further) development and exploitation of advanced diagnostics is needed to derive a seamless tomography of the atmosphere as an integral phenomenon.

Until we succeed to reach these ambitious goals, we are left with a number of open questions. Of particular interest for the quiet Sun are, amongst others:

- How does the weak internetwork field connect with the stronger network field? What does the magnetic field look like just below the "canopy"? And can we talk about a "canopy" even in a wider sense after all?

- How do the propagating fluctospheric shock waves interact with the stronger ("canopy") field? Is it mostly a "passive" refraction/reflection at the "boundaries" of flux concentrations (e.g., Rosenthal et al. 2002; Steiner et al. 2007) or also "active" distortion/displacement/compression of magnetic field? How and where does mode conversion take exactly place under realistic conditions?

- The question of the coupling between the atmospheric layers is closely connected to the heating mechanism question or, better said, to the question of the atmospheric energy balance, not only for the Sun but also for other stars. Amongst other things, the ongoing controversy concerning the heating mechanism of the quiet Sun chromosphere (e.g., Fossum and Carlsson 2005) has important implications for stellar activity in general.

Acknowledgements We would like to thank O. Steiner, K. Schrijver, B. de Pontieu, M. Carlsson, V. Hansteen, R. Rutten, H. Peter, and $\varnothing$. Langangen for helpful discussions. SWB was supported with a Marie Curie Intra-European Fellowship of the European Commission (6th Framework Programme, FP62005-Mobility-5, Proposal No. 042049). Å.N. acknowledges support from the Danish Natural Science Research Council and from the Danish Center for Scientific Computing.

\section{References}

W.P. Abbett, The magnetic connection between the convection zone and corona in the quiet Sun. Astrophys. J. 665, 1469-1488 (2007)

T. Amari, J.J. Aly, J.F. Luciani, T.Z. Boulmezaoud, Z. Mikic, Reconstructing the solar coronal magnetic field as a force-free magnetic field. Sol. Phys. 174, 129-149 (1997)

A. Asensio Ramos, J. Trujillo Bueno, E. Landi Degl'Innocenti, Advanced forward modeling and inversion of stokes profiles resulting from the joint action of the Hanle and Zeeman effects. Astrophys. J. 683, 542-565 (2008)

M. Asplund, Å. Nordlund, R. Trampedach, C. Allende Prieto, R.F. Stein, Line formation in solar granulation. I. Fe line shapes, shifts and asymmetries. Astron. Astrophys. 359, 729-742 (2000a)

M. Asplund, H.-G. Ludwig, A. Nordlund, R.F. Stein, The effects of numerical resolution on hydrodynamical surface convection simulations and spectral line formation. Astron. Astrophys. 359, 669-681 (2000b)

R.G. Athay, Are spicules related to coronal heating? Sol. Phys. 197, 31-42 (2000)

T.R. Ayres, Does the Sun have a full-time COmosphere? Astrophys. J. 575, 1104-1115 (2002) 
T.E. Berger, B. De Pontieu, L. Fletcher, C.J. Schrijver, T.D. Tarbell, A.M. Title, What is moss? Sol. Phys. 190, 409-418 (1999)

M. Bianda, J.O. Stenflo, S.K. Solanki, Hanle diagnostics of solar magnetic fields: the SR II 4078 Angstrom line. Astron. Astrophys. 337, 565-578 (1998)

T.J. Bogdan, M. Carlsson, V.H. Hansteen, A. McMurry, C.S. Rosenthal, M. Johnson, S. Petty-Powell, E.J. Zita, R.F. Stein, S.W. McIntosh, A. Nordlund, Waves in the magnetized solar atmosphere. II. Waves from localized sources in magnetic flux concentrations. Astrophys. J. 599, 626-660 (2003)

V. Bommier, Quantum theory of the Hanle effect. II-Effect of level-crossings and anti-level-crossings on the polarization of the D3 helium line of solar prominences. Astron. Astrophys. 87, 109-120 (1980)

P.S. Cally, What to look for in the seismology of solar active regions. Astron. Nachr. 328, 286 (2007)

M. Carlsson, A computer program for solving multi-level non-LTE radiative transfer problems in moving or static atmospheres. Report No. 33, Uppsala Astronomical Observatory

M. Carlsson, Modeling the Solar Chromosphere, in The Physics of Chromospheric Plasmas, ed. by P. Heinzel, I. Dorotovič, R.J. Rutten. Astronomical Society of the Pacific Conference Series, vol. 368 (ASP, San Francisco, 2007), p. 49

M. Carlsson, R.F. Stein, in Proc. Mini-Workshop on Chromospheric Dynamics, ed. by M. Carlsson (Inst. Theor. Astrophys., Oslo, 1994), p. 47

M. Carlsson, R.F. Stein, Does a nonmagnetic solar chromosphere exist? Astrophys. J. Lett. 440, L29-L32 (1995)

M. Carlsson, R.F. Stein, Formation of solar calcium H and K bright grains. Astrophys. J. 481, 500 (1997)

M. Carlsson, R.F. Stein, Dynamic hydrogen ionization. Astrophys. J. 572, 626-635 (2002)

R. Casini, A. López Ariste, S. Tomczyk, B.W. Lites, Magnetic maps of prominences from full stokes analysis of the He I D3 line. Astrophys. J. Lett. 598, L67-L70 (2003)

G. Cauzzi, K.P. Reardon, H. Uitenbroek, F. Cavallini, A. Falchi, R. Falciani, K. Janssen, T. Rimmele, A. Vecchio, F. Wöger, The solar chromosphere at high resolution with IBIS. I. New insights from the Ca II $854.2 \mathrm{~nm}$ line. Astron. Astrophys. 480, 515-526 (2008)

R. Centeno, H. Socas-Navarro, B. Lites, M. Kubo, Z. Frank, R. Shine, T. Tarbell, A. Title, K. Ichimoto, S. Tsuneta, Y. Katsukawa, Y. Suematsu, T. Shimizu, S. Nagata, Emergence of small-scale magnetic loops in the quiet-Sun internetwork. Astrophys. J. Lett. 666, L137-L140 (2007)

M.C.M. Cheung, M. Schüssler, F. Moreno-Insertis, Magnetic flux emergence in granular convection: radiative MHD simulations and observational signatures. Astron. Astrophys. 467, 703-719 (2007)

M. Collados, I. Rodríguez Hidalgo, L. Bellot Rubio, B. Ruiz Cobo, D. Soltau, TIP (Tenerife Infrared Polarimeter): A near IR full Stokes polarimeter for the German solar telescopes at Observatorio del Teide, in Astronomische Gesellschaft Meeting Abstracts (1999), p. 13

M. Collados, A. Lagg, J. Díaz García, E. Hernández Suárez, R. López López, E. Páez Mañá, S.K. Solanki, Tenerife Infrared Polarimeter II, in The Physics of Chromospheric Plasmas, ed. by P. Heinzel, I. Dorotovič, R.J. Rutten. ASP Conference Series, vol. 368 (ASP, San Francisco, 2007), pp. 611-616

S. Danilovic, A. Gandorfer, A. Lagg, M. Schüssler, S.K. Solanki, A. Vögler, Y. Katsukawa, S. Tsuneta, The intensity contrast of solar granulation: comparing Hinode SP results with MHD simulations. Astron. Astrophys. 484, L17-L20 (2008)

B. De Pontieu, T. Tarbell, R. Erdélyi, Correlations on arcsecond scales between chromospheric and transition region emission in active regions. Astrophys. J. 590, 502-518 (2003)

B. De Pontieu, R. Erdélyi, S.P. James, Solar chromospheric spicules from the leakage of photospheric oscillations and flows. Nature 430, 536-539 (2004)

B. De Pontieu, S. McIntosh, V.H. Hansteen, M. Carlsson, C.J. Schrijver, T.D. Tarbell, A.M. Title, R.A. Shine, Y. Suematsu, S. Tsuneta, Y. Katsukawa, K. Ichimoto, T. Shimizu, S. Nagata, A tale of two spicules: the impact of spicules on the magnetic chromosphere. Publ. Astron. Soc. Jpn. 59, 655 (2007a)

B. De Pontieu, S.W. McIntosh, M. Carlsson, V.H. Hansteen, T.D. Tarbell, C.J. Schrijver, A.M. Title, R.A. Shine, S. Tsuneta, Y. Katsukawa, K. Ichimoto, Y. Suematsu, T. Shimizu, S. Nagata, Chromospheric alfvénic waves strong enough to power the solar wind. Science 318, 1574 (2007b)

B. De Pontieu, V.H. Hansteen, L. Rouppe van der Voort, M. van Noort, M. Carlsson, High-resolution observations and numerical simulations of chromospheric fibrils and mottles, in The Physics of Chromospheric Plasmas, ed. by P. Heinzel, I. Dorotovič, R.J. Rutten. Astronomical Society of the Pacific Conference Series, vol. 368 (ASP, San Francisco, 2007c), p. 65

A.G. de Wijn, B.W. Lites, T.E. Berger, Z.A. Frank, T.D. Tarbell, R. Ishikawa, Hinode observations of magnetic elements in internetwork areas. Astrophys. J. 684, 1469-1476 (2008)

P. Dmitruk, D.O. Gómez, Scaling law for the heating of solar coronal loops. Astrophys. J. Lett. 527, L63-L66 (1999)

S.B.F. Dorch, Å. Nordlund, On the transport of magnetic fields by solar-like stratified convection. Astron. Astrophys. 365, 562-570 (2001) 
D. Dravins, Å. Nordlund, Stellar granulation, Part Four-Line formation in inhomogeneous stellar photospheres. Astron. Astrophys. 228, 184 (1990)

D. Dravins, L. Lindegren, A. Nordlund, Solar granulation-Influence of convection on spectral line asymmetries and wavelength shifts. Astron. Astrophys. 96, 345-364 (1981)

W. Finsterle, S.M. Jefferies, A. Cacciani, P. Rapex, S.W. McIntosh, Helioseismic mapping of the magnetic canopy in the solar chromosphere. Astrophys. J. Lett. 613, L185-L188 (2004)

L. Fletcher, B. De Pontieu, Plasma diagnostics of transition region "moss" using SOHO/CDS and TRACE. Astrophys. J. Lett. 520, L135-L138 (1999)

J.M. Fontenla, E.H. Avrett, R. Loeser, Energy balance in the solar transition region. III-Helium emission in hydrostatic, constant-abundance models with diffusion. Astrophys. J. 406, 319-345 (1993)

A. Fossum, M. Carlsson, High-frequency acoustic waves are not sufficient to heat the solar chromosphere. Nature 435, 919-921 (2005)

B. Freytag, M. Steffen, B. Dorch, Spots on the surface of Betelgeuse-Results from new 3D stellar convection models. Astron. Nachr. 323, 213-219 (2002)

A.H. Gabriel, A magnetic model of the solar transition region. R. Soc. Lond. Philos. Trans. Ser. A 281, 339-352 (1976)

D.J. Galloway, N.O. Weiss, Convection and magnetic fields in stars. Astrophys. J. 243, 945-953 (1981)

K. Galsgaard, A. Nordlund, Heating and activity of the solar corona 1. Boundary shearing of an initially homogeneous magnetic field. J. Gephys. Res. 101, 13445-13460 (1996)

D. Georgobiani, J. Zhao, A.G. Kosovichev, D. Benson, R.F. Stein, Å. Nordlund, Local helioseismology and correlation tracking analysis of surface structures in realistic simulations of solar convection. Astrophys. J. 657, 1157-1161 (2007)

R.G. Giovanelli, H.P. Jones, The three-dimensional structure of atmospheric magnetic fields in two active regions. Sol. Phys. 79, 267-278 (1982)

B.V. Gudiksen, Connections: Photosphere-chromosphere-corona, in Solar MHD Theory and Observations: A High Spatial Resolution Perspective, ed. by J. Leibacher, R.F. Stein, H. Uitenbroek. Astronomical Society of the Pacific Conference Series, vol. 354 (ASP, San Francisco, 2006), p. 331

B.V. Gudiksen, Å. Nordlund, Bulk heating and slender magnetic loops in the solar corona. Astrophys. J. Lett. 572, L113-L116 (2002)

B.V. Gudiksen, Å. Nordlund, An ab initio approach to solar coronal loops. Astrophys. J. 618, 1031-1038 (2005a)

B.V. Gudiksen, Å. Nordlund, An ab initio approach to the solar coronal heating problem. Astrophys. J. 618, 1020-1030 (2005b)

G.E. Hale, On the probable existence of a magnetic field in Sun-spots. Astrophys. J. 28, 315 (1908)

W. Hanle, Über magnetische beeinflussung der polarisation der resonanzfluoreszenz. Z. Phys. D: At. Mol. Clust. 18(1), 5-10 (1991). Reprinted from Z. Phys. 30, 93 (1924). ISSN 0178-7683 (Print) 1431-5866 (Online)

V.H. Hansteen, Waves and shocks in the solar atmosphere, in New Solar Physics with Solar-B Mission, ed. by K. Shibata, S. Nagata, T. Sakurai. Astronomical Society of the Pacific Conference Series, vol. 369 (ASP, San Francisco, 2007), p. 193

V.H. Hansteen, B. Gudiksen, 3D numerical models of quiet Sun coronal heating, in Solar Wind 11/SOHO 16, Connecting Sun and Heliosphere. ESA Special Publication, vol. 592 (ESA, Noordwijk, 2005)

V.H. Hansteen, B. De Pontieu, L. Rouppe van der Voort, M. van Noort, M. Carlsson, Dynamic fibrils are driven by magnetoacoustic shocks. Astrophys. J. Lett. 647, L73-L76 (2006)

V.H. Hansteen, M. Carlsson, B. Gudiksen, 3D numerical models of the chromosphere, transition region, and corona, in The Physics of Chromospheric Plasmas, ed. by P. Heinzel, I. Dorotovič, R.J. Rutten. Astronomical Society of the Pacific Conference Series, vol. 368 (ASP, San Francisco, 2007), p. 107

R.A. Harrison, A statistical study of the coronal mass ejection phenomenon. Adv. Space Res. 14, 23 (1994)

S.S. Hasan, O. Steiner, A. van Ballegooijen, Inferring the chromospheric magnetic topology through waves, in IAU Symposium, vol. 247 (2008), pp. 78-81

D.L. Hendrix, G. van Hoven, Z. Mikic, D.D. Schnack, The viability of ohmic dissipation as a coronal heating source. Astrophys. J. 470, 1192 (1996)

R. Ishikawa, S. Tsuneta, K. Ichimoto, H. Isobe, Y. Katsukawa, B.W. Lites, S. Nagata, T. Shimizu, R.A. Shine, Y. Suematsu, T.D. Tarbell, A.M. Title, Transient horizontal magnetic fields in solar plage regions. Astron. Astrophys. 481, L25-L28 (2008)

S.M. Jefferies, S.W. McIntosh, J.D. Armstrong, T.J. Bogdan, A. Cacciani, B. Fleck, Magnetoacoustic portals and the Basal heating of the solar chromosphere. Astrophys. J. Lett. 648, L151-L155 (2006)

S. Jendersie, H. Peter, Link between the chromospheric network and magnetic structures of the corona. Astron. Astrophys. 460, 901-908 (2006)

H.P. Jones, R.G. Giovanelli, Magnetograph response to canopy-type fields. Sol. Phys. 79, 247-266 (1982) 
P. Judge, Observations of the solar chromosphere, in Solar MHD Theory and Observations: A High Spatial Resolution Perspective, ed. by J. Leibacher, R.F. Stein, H. Uitenbroek. Astronomical Society of the Pacific Conference Series, vol. 354 (ASP, San Francisco, 2006), p. 259

L. Kleint, K. Reardon, J.O. Stenflo, H. Uitenbroek, A. Tritschler, Spectropolarimetry of Ca II 8542: probing the chromospheric magnetic field, in Proceedings of the 5th Solar Polarization Workshop, Ascona, Switzerland, 17-21 September 2007, ed. by N.K.N.S. Berdyugina, R. Ramelli (2008)

J.M. Krijger, R.J. Rutten, B.W. Lites, T. Straus, R.A. Shine, T.D. Tarbell, Dynamics of the solar chromosphere. III. Ultraviolet brightness oscillations from TRACE. Astron. Astrophys. 379, 1052-1082 (2001)

A. Lagg, L. Merenda, Measurement of the magnetic canopy over supergranulation cell (2008, in preparation)

A. Lagg, J. Woch, N. Krupp, S.K. Solanki, Retrieval of the full magnetic vector with the He I multiplet at $1083 \mathrm{~nm}$. Maps of an emerging flux region. Astron. Astrophys. 414, 1109-1120 (2004)

A. Lagg, J. Woch, S. Solanki, N. Krupp, Supersonic downflows in the vicinity of a growing pore: Evidence of unresolved magnetic fine structure at chromospheric heights. Astron. Astrophys. 462, 1147-1155 (2007)

E. Landi Degl'Innocenti, The determination of vector magnetic fields in prominences from the observations of the Stokes profiles in the D3 line of Helium. Sol. Phys. 79, 291-322 (1982)

$\varnothing$. Langangen, L. Rouppe van der Voort, Y. Lin, Measurements of plasma motions in dynamic fibrils. Astrophys. J. 673, 1201-1208 (2008a)

Ø. Langangen, B. De Pontieu, M. Carlsson, V.H. Hansteen, G. Cauzzi, K. Reardon, Search for high velocities in the disk counterpart of type II spicules. Astrophys. J. Lett. 679, L167-L170 (2008b)

$\varnothing$. Langangen, M. Carlsson, L. Rouppe van der Voort, V. Hansteen, B. De Pontieu, Spectroscopic measurements of dynamic fibrils in the Ca II $\lambda=8662$ line. Astrophys. J. 673, 1194-1200 (2008c)

J. Lee, Radio emissions from solar active regions. Space Sci. Rev. 133, 73-102 (2007)

J. Leenaarts, S. Wedemeyer-Böhm, Time-dependent hydrogen ionisation in 3D simulations of the solar chromosphere. Methods and first results. Astron. Astrophys. 460, 301-307 (2006)

J. Leenaarts, M. Carlsson, V. Hansteen, R.J. Rutten, Non-equilibrium hydrogen ionization in 2D simulations of the solar atmosphere. Astron. Astrophys. 473, 625-632 (2007)

B.W. Lites, K.D. Leka, A. Skumanich, V. Martinez Pillet, T. Shimizu, Small-scale horizontal magnetic fields in the solar photosphere. Astrophys. J. 460, 1019 (1996)

B.W. Lites, R.J. Rutten, T.E. Berger, Dynamics of the solar chromosphere. II. Ca II H_2V and K_2V grains versus internetwork fields. Astrophys. J. 517, 1013-1033 (1999)

B. Lites, H. Socas-Navarro, M. Kubo, T.E. Berger, Z. Frank, R.A. Shine, T.D. Tarbell, A.M. Title, K. Ichimoto, Y. Katsukawa, S. Tsuneta, Y. Suematsu, T. Shimizu, S. Nagata, Hinode observations of horizontal quiet Sun magnetic flux and the "hidden turbulent magnetic flux". Publ. Astron. Soc. Jpn. 59, 571 (2007)

B.W. Lites, M. Kubo, H. Socas-Navarro, T. Berger, Z. Frank, R. Shine, T. Tarbell, A. Title, K. Ichimoto, Y. Katsukawa, S. Tsuneta, Y. Suematsu, T. Shimizu, S. Nagata, The horizontal magnetic flux of the quiet-Sun internetwork as observed with the hinode spectro-polarimeter. Astrophys. J. 672, 1237-1253 (2008)

A. López Ariste, R. Casini, Inference of the magnetic field in spicules from spectropolarimetry of He I D3. Astron. Astrophys. 436, 325-331 (2005)

M.A. Loukitcheva, S.K. Solanki, S. White, ALMA as the ideal probe of the solar chromosphere. Astrophys. Space Sci. 313, 197-200 (2008)

M.J. Martínez González, M. Collados, B. Ruiz Cobo, C. Beck, Internetwork magnetic field distribution from simultaneous $1.56 \mu \mathrm{m}$ and $630 \mathrm{~nm}$ observations. Astron. Astrophys. 477, 953-965 (2008)

J. Martínez-Sykora, V. Hansteen, M. Carlsson, Twisted flux tube emergence from the convection zone to the corona. Astrophys. J. 679, 871-888 (2008)

S.W. McIntosh, P.G. Judge, On the nature of magnetic shadows in the solar chromosphere. Astrophys. J. 561, 420-426 (2001)

S.W. McIntosh, T.J. Bogdan, P.S. Cally, M. Carlsson, V.H. Hansteen, P.G. Judge, B.W. Lites, H. Peter, C.S. Rosenthal, T.D. Tarbell, An observational manifestation of magnetoatmospheric waves in internetwork regions of the chromosphere and transition region. Astrophys. J. Lett. 548, L237-L241 (2001)

L. Merenda, J. Trujillo Bueno, M. Collados, A magnetic map of a solar filament, in The Physics of Chromospheric Plasmas, ed. by P. Heinzel, I. Dorotovič. Astronomical Society of the Pacific Conference Series, vol. 368 (ASP, San Francisco, 2007), p. 347

Å. Nordlund, 3-D Model calculations, in Small Scale Magnetic Flux Concentrations in the Solar Photosphere, ed. by W. Deinzer, M. Knölker, H.H. Voigt (1986), p. 83

Å. Nordlund, D. Dravins, Stellar granulation. III-Hydrodynamic model atmospheres. IV-Line formation in inhomogeneous stellar photospheres. V-Synthetic spectral lines in disk-integrated starlight. Astron. Astrophys. 228, 155-217 (1990) 
T.J. Okamoto, S. Tsuneta, T.E. Berger, K. Ichimoto, Y. Katsukawa, B.W. Lites, S. Nagata, K. Shibata, T. Shimizu, R.A. Shine, Y. Suematsu, T.D. Tarbell, A.M. Title, Coronal transverse magnetohydrodynamic waves in a solar prominence. Science 318, 1577 (2007)

D. Orozco Suárez, L.R. Bellot Rubio, J.C. del Toro Iniesta, S. Tsuneta, B.W. Lites, K. Ichimoto, Y. Katsukawa, S. Nagata, T. Shimizu, R.A. Shine, Y. Suematsu, T.D. Tarbell, A.M. Title, Quiet-Sun internetwork magnetic fields from the inversion of hinode measurements. Astrophys. J. Lett. 670, L61-L64 (2007)

E.N. Parker, Topological dissipation and the small-scale fields in turbulent gases. Astrophys. J. 174, 499 (1972)

E.N. Parker, The dissipation of inhomogeneous magnetic fields and the problem of coronae. I-Dislocation and flattening of flux tubes. II-The dynamics of dislocated flux. Astrophys. J. 244, 631-652 (1981)

E.N. Parker, Magnetic neutral sheets in evolving fields-Part two-Formation of the solar corona. Astrophys. J. 264, 642 (1983)

H. Peter, B.V. Gudiksen, Å. Nordlund, Coronal heating through braiding of magnetic field lines. Astrophys. J. Lett. 617, L85-L88 (2004)

H. Peter, B.V. Gudiksen, A. Nordlund, Forward modeling of the corona of the Sun and solar-like Stars: From a three-dimensional magnetohydrodynamic model to synthetic extreme-ultraviolet spectra. Astrophys. J. 638, 1086-1100 (2006)

A. Pietarila, J. Hirzberger, S. Solanki, Fibrils in cak. Astron. Astrophys. (2008, in preparation)

C.S. Rosenthal, T.J. Bogdan, M. Carlsson, S.B.F. Dorch, V. Hansteen, S.W. McIntosh, A. McMurry, Å. Nordlund, R.F. Stein, Waves in the magnetized solar atmosphere. I. Basic processes and internetwork oscillations. Astrophys. J. 564, 508-524 (2002)

L.H.M. Rouppe van der Voort, V.H. Hansteen, M. Carlsson, A. Fossum, E. Marthinussen, M.J. van Noort, T.E. Berger, Solar magnetic elements at 0.1 arcsec resolution. II. Dynamical evolution. Astron. Astrophys. 435, 327-337 (2005)

L.H.M. Rouppe van der Voort, B. De Pontieu, V.H. Hansteen, M. Carlsson, M. van Noort, Magnetoacoustic shocks as a driver of quiet-Sun mottles. Astrophys. J. Lett. 660, L169-L172 (2007)

R.J. Rutten, On the nature of the solar chromosphere, in Solar MHD Theory and Observations: A High Spatial Resolution Perspective, ed. by J. Leibacher, R.F. Stein, H. Uitenbroek. Astronomical Society of the Pacific Conference Series, vol. 354 (ASP, San Francisco, 2006), p. 276

R.J. Rutten, Observing the solar chromosphere, in The Physics of Chromospheric Plasmas, ed. by P. Heinzel, I. Dorotovič, R.J. Rutten. Astronomical Society of the Pacific Conference Series, vol. 368 (ASP, San Francisco, 2007), p. 27

T. Sakurai, Calculation of force-free magnetic field with non-constant $\alpha$. Sol. Phys. 69, 343-359 (1981)

T. Sakurai, Computational modeling of magnetic fields in solar active regions. Space Sci. Rev. 51, 11-48 (1989)

C. Sasso, A. Lagg, S.K. Solanki, R. Aznar Cuadrado, M. Collados, Full-stokes observations and analysis of He I $10830 \AA$ in a flaring region, in The Physics of Chromospheric Plasmas, ed. by P. Heinzel, I. Dorotovič R.J. Rutten. Astronomical Society of the Pacific Conference Series, vol. 368 (ASP, San Francisco, 2007), p. 467

W. Schaffenberger, S. Wedemeyer-Böhm, O. Steiner, B. Freytag, Magnetohydrodynamic simulation from the convection zone to the chromosphere, in Chromospheric and Coronal Magnetic Fields, ed. by D.E. Innes, A. Lagg, S.A. Solanki. ESA Special Publication, vol. 596 (ESA, Noordwijk, 2005)

W. Schaffenberger, S. Wedemeyer-Böhm, O. Steiner, B. Freytag, Holistic MHD-simulation from the convection zone to the chromosphere, in Solar MHD Theory and Observations: A High Spatial Resolution Perspective, ed. by J. Leibacher, R.F. Stein, H. Uitenbroek. Astronomical Society of the Pacific Conference Series, vol. 354 (ASP, San Francisco, 2006), p. 345

G.B. Scharmer, G. Narayan, T. Hillberg, J. de la Cruz Rodriguez, M.G. Lofdahl, D. Kiselman, P. Sutterlin, M. van Noort, A. Lagg, CRISP Spectropolarimetric Imaging of Penumbral Fine Structure. ArXiv e-prints, 806 (2008)

C.J. Schrijver, A.M. Title, The magnetic connection between the solar photosphere and the corona. Astrophys. J. Lett. 597, L165-L168 (2003)

C.J. Schrijver, A.A. van Ballegooijen, Is the quiet-Sun corona a quasi-steady, force-free environment? Astrophys. J. 630, 552-560 (2005)

M. Schüssler, A. Vögler, Strong horizontal photospheric magnetic field in a surface dynamo simulation. Astron. Astrophys. 481, L5-L8 (2008)

R. Skartlien, R.F. Stein, A. Nordlund, Excitation of chromospheric wave transients by collapsing granules. Astrophys. J. 541, 468-488 (2000)

H. Socas-Navarro, D. Elmore, Physical properties of spicules from simultaneous spectropolarimetric observations of He I and Ca II lines. Astrophys. J. Lett. 619, L195-L198 (2005) 
H. Socas-Navarro, D. Elmore, A. Pietarila, A. Darnell, B.W. Lites, S. Tomczyk, S. Hegwer, Spinor: visible and infrared spectro-polarimetry at the national solar observatory. Sol. Phys. 235, 55-73 (2006)

S.K. Solanki, A. Lagg, J. Woch, N. Krupp, M. Collados, Three-dimensional magnetic field topology in a region of solar coronal heating. Nature 425, 692-695 (2003)

R.F. Stein, Å. Nordlund, Simulations of solar granulation. I. General properties. Astrophys. J. 499, 914 (1998)

R.F. Stein, Å. Nordlund, Solar small-scale magnetoconvection. Astrophys. J. 642, 1246-1255 (2006)

R.F. Stein, D. Benson, D. Georgobiani, A. Nordlund, Supergranule scale convection simulations, in Proceedings of SOHO 18/GONG 2006/HELAS I, Beyond the Spherical Sun. ESA Special Publication, vol. 624 (ESA, Noordwijk, 2006)

O. Steiner, Distribution of magnetic flux density at the solar surface. Formulation and results from simulations. Astron. Astrophys. 406, 1083-1088 (2003)

O. Steiner, Recent progresses in the simulation of small-scale magnetic fields, in Modern Solar FacilitiesAdvanced Solar Science, ed. by F. Kneer, K.G. Puschmann, A.D. Wittmann (2007), p. 321

O. Steiner, P. Murdin, Chromosphere: Magnetic canopy, in Encyclopedia of Astronomy and Astrophysics (2000)

O. Steiner, U. Grossmann-Doerth, M. Knoelker, M. Schuessler, Dynamical interaction of solar magnetic elements and granular convection: Results of a numerical simulation. Astrophys. J. 495, 468 (1998)

O. Steiner, G. Vigeesh, L. Krieger, S. Wedemeyer-Böhm, W. Schaffenberger, B. Freytag, First local helioseismic experiments with $\mathrm{CO}^{5}$ BOLD. Astron. Nachr. 328, 323 (2007)

O. Steiner, R. Rezaei, W. Schaffenberger, S. Wedemeyer-Böhm, The horizontal internetwork magnetic field: Numerical simulations in comparison to observations with hinode. Astrophys. J. Lett. 680, L85-L88 (2008)

J.O. Stenflo, R. Holzreuter, Empirical view of magnetoconvection, in SOLMAG 2002. Proceedings of the Magnetic Coupling of the Solar Atmosphere Euroconference, ed. by H. Sawaya-Lacoste. ESA Special Publication, vol. 505 (ESA, Noordwijk, 2002), pp. 101-104

J.O. Stenflo, R. Holzreuter, Flux tubes or fractal distributions - on the nature of photospheric magnetic fields. Astron. Nachr. 324, 397 (2003)

J.O. Stenflo, C.U. Keller, The second solar spectrum. A new window for diagnostics of the Sun. Astron. Astrophys. 321, 927-934 (1997)

J.O. Stenflo, A. Gandorfer, R. Holzreuter, D. Gisler, C.U. Keller, M. Bianda, Spatial mapping of the Hanle and Zeeman effects on the Sun. Astron. Astrophys. 389, 314-324 (2002)

T. Straus, B. Fleck, S. Jefferies, G. Cauzzi, C. G., S. McIntosh, K. Reardon, G. Severino, M. Steffen, The energy flux of internal gravity waves in the lower solar atmosphere. Astrophys. J. Lett. 681, L125-L128 (2008)

Y. Suematsu, Y. Katsukawa, K. Ichimoto, S. Tsuneta, T. Okamoto, S. Nagata, T. Shimizu, T. Tarbell, R. Shine, A. Title, High resolution observation of spicules in Ca II H with Hinode/SOT, in American Astronomical Society Meeting Abstracts, vol. 210 (2007), p. 94.11

S.M. Tobias, N.H. Brummell, T.L. Clune, J. Toomre, Pumping of magnetic fields by turbulent penetrative convection. Astrophys. J. Lett. 502, L177 (1998)

S.M. Tobias, N.H. Brummell, T.L. Clune, J. Toomre, Transport and storage of magnetic field by overshooting turbulent compressible convection. Astrophys. J. 549, 1183-1203 (2001)

J. Trujillo Bueno, E. Landi Degl'Innocenti, M. Collados, L. Merenda, R. Manso Sainz, Selective absorption processes as the origin of puzzling spectral line polarization from the Sun. Nature 415, 403-406 (2002)

J. Trujillo Bueno, N. Shchukina, A. Asensio Ramos, A substantial amount of hidden magnetic energy in the quiet Sun. Nature 430, 326-329 (2004)

J. Trujillo Bueno, L. Merenda, R. Centeno, M. Collados, E. Landi Degl'Innocenti, The Hanle and Zeeman effects in solar spicules: A novel diagnostic window on chromospheric magnetism. Astrophys. J. Lett. 619, L191-L194 (2005)

A. Vögler, M. Schüssler, A solar surface dynamo. Astron. Astrophys. 465, L43-L46 (2007)

A. Vögler, S. Shelyag, M. Schüssler, F. Cattaneo, T. Emonet, T. Linde, Simulations of magneto-convection in the solar photosphere. Equations, methods, and results of the MURaM code. Astron. Astrophys. 429, 335-351 (2005)

S. Wedemeyer, Multi-dimensional radiation hydrodynamic simulations of the non-magnetic solar atmosphere. Ph.D. thesis, University of Kiel, 2003. http://e-diss.uni-kiel.de/diss_764/

S. Wedemeyer, B. Freytag, M. Steffen, H.-G. Ludwig, H. Holweger, Numerical simulation of the threedimensional structure and dynamics of the non-magnetic solar chromosphere. Astron. Astrophys. 414, 1121-1137 (2004)

S. Wedemeyer-Böhm, Point spread functions for the Solar optical telescope onboard Hinode. Astron. Astrophys. 487, 399-412 (2008)

S. Wedemeyer-Böhm, M. Steffen, Carbon monoxide in the solar atmosphere. II. Radiative cooling by CO lines. Astron. Astrophys. 462, L31-L35 (2007) 
S. Wedemeyer-Böhm, F. Wöger, Small-scale structure and dynamics of the lower solar atmosphere, in IAU Symposium, vol. 247 (2008), pp. 66-73

S. Wedemeyer-Böhm, I. Kamp, J. Bruls, B. Freytag, Carbon monoxide in the solar atmosphere. I. Numerical method and two-dimensional models. Astron. Astrophys. 438, 1043-1057 (2005a)

S. Wedemeyer-Böhm, W. Schaffenberger, O. Steiner, M. Steffen, B. Freytag, I. Kamp, Simulations of magnetohydrodynamics and $\mathrm{CO}$ formation from the convection zone to the chromosphere, in Chromospheric and Coronal Magnetic Fields, ed. by D.E. Innes, A. Lagg, S.A. Solanki. ESA Special Publication, vol. 596 (ESA, Noordwijk, 2005b)

S. Wedemeyer-Böhm, I. Kamp, B. Freytag, J. Bruls, M. Steffen, A first three-dimensional model for the carbon monoxide concentration in the solar atmosphere, in Solar MHD Theory and Observations: A High Spatial Resolution Perspective, ed. by J. Leibacher, R.F. Stein, H. Uitenbroek. Astronomical Society of the Pacific Conference Series, vol. 354 (ASP, San Francisco, 2006), p. 301

S. Wedemeyer-Böhm, H.G. Ludwig, M. Steffen, J. Leenaarts, B. Freytag, Inter-network regions of the Sun at millimetre wavelengths. Astron. Astrophys. 471, 977-991 (2007)

N.O. Weiss, D.P. Brownjohn, P.C. Matthews, M.R.E. Proctor, Photospheric convection in strong magnetic fields. Mon. Not. R. Astron. Soc. 283, 1153-1164 (1996)

T. Wiegelmann, Nonlinear force-free modeling of the solar coronal magnetic field. J. Geophys. Res. (Space Phys.) 113(A12), 3 (2008)

T. Wiegelmann, J.K. Thalmann, C.J. Schrijver, M.L. Derosa, T.R. Metcalf, Can we improve the preprocessing of photospheric vector magnetograms by the inclusion of chromospheric observations? Sol. Phys. 247, 249-267 (2008)

F. Wöger, S. Wedemeyer-Böhm, W. Schmidt, O. von der Lühe, Observation of a short-lived pattern in the solar chromosphere. Astron. Astrophys. 459, L9-L12 (2006)

J. Zhao, D. Georgobiani, A.G. Kosovichev, D. Benson, R.F. Stein, Å. Nordlund, Validation of time-distance helioseismology by use of realistic simulations of solar convection. Astrophys. J. 659, 848-857 (2007) 